Journal of Mathematics and Statistics 6 (2): 193-204, 2010

ISSN 1549-3644

(C) 2010 Science Publications

\title{
Higher-Order Newton-Cotes Formulas
}

\author{
Pedro Americo Almeida Magalhaes Junior and Cristina Almeida Magalhaes \\ Pontificia Universidade Catolica de Minas Gerais, Av. Dom Jose Gaspar 500, 30535-610, \\ Belo Horizonte, Minas Gerais, Brazil
}

\begin{abstract}
Problem statement: The present work offers equations of Newton-Cotes Integration until twenty segments. Approach: It shows Newton-Cotes closed and open integration formulas. The new type Newton-Cotes semi-closed or semi-open was proposed. Results: An analysis of error in the technique was made. To estimate the error of integration in discrete data, we propose apply different rules or mix several rules. Conclusion/Recommendations: The difference in the result of each formula provides an approximation of the error. Computational routines to generate Newton-Cotes integration rules were presented.
\end{abstract}

Key words: Numerical methods, numerical integration, quadrature, Newton-cotes, higher-order, analysis of error

\section{INTRODUCTION}

The evaluation of integrals, a process known as integration or quadrature, is required in many problems in engineering and science (Sermutlu, 2005). The function $\mathrm{f}(\mathrm{x})$, which is to be integrated, may be a known function or a set of discrete data. Some known functions have an exact integral, in which case can be evaluated exactly in closed form. Many known functions, however, do not have an exact integral and an approximate numerical procedure is required to evaluate. In many cases, the function $\mathrm{f}(\mathrm{x})$ is known only at a set of discrete points, in which case an approximate numerical procedure is again required to evaluate (Simos, 2008). Numerical integration (quadrature) formulas can be developed by fitting approximating functions (e.g., polynomials) to discrete data and integrating the approximating function:

$I=\int_{x_{1}}^{x_{N}} f(x) d x \cong \int_{x_{1}}^{x_{1}+(N-1) h} P(x) d x$

When the function to be integrated is known at equally spaced points $(\Delta \mathrm{x}=\mathrm{h}=$ constant $)$ and $\mathrm{N}$ is number of points with $x$ ranging $x_{1}, x_{1}+h, x_{1}+2 h, \ldots$, $\mathrm{x}_{1}+(\mathrm{N}-1) \mathrm{h}$. The polynomial can be fit to the discrete data with much less effort, thus significantly decreasing the amount of effort required (Simos, 2008). The resulting formulas are called Newton-cotes formulas.

The distance between the lower and upper limits of integration is called the range of integration. The distance between any two data points is called an increment $(\Delta x=h)$. A linear polynomial requires one increment and two data points to obtain a fit. A quadratic polynomial requires two increments and three data points to obtain a fit. And so on for higher-degree polynomials. The group of increments required to fit a polynomial is called an interval (Kalogiratou and Simos, 2003). A linear polynomial requires an interval consisting of only one increment. A quadratic polynomial requires an interval containing two increments. And so on. The total range of integration can consist of one or more intervals. Each interval consists of one or more increments, depending on the degree of the approximating polynomial.

Closed and open forms of Newton-Cotes formulas are available. The closed forms are those where the data points at the beginning and end of the limits of integration are known. The open forms have integration limits that extend beyond the range of the data (Witteveen et al., 2009).

\section{MATERIALS AND METHODS}

Newton-cotes closed integration equation: The rule for a single interval is obtained by fitting a first-degree polynomial to two discrete points (Sermutlu and Eyyubog'lu, 2007). The upper limit of integration is $\mathrm{x}_{2}=\mathrm{x}_{1}+\mathrm{h}$, then the integral (I) have the formula:

$$
\mathrm{I}=\frac{\mathrm{h}}{2}\left(\mathrm{y}_{1}+\mathrm{y}_{2}\right) \quad \text { or } \quad \mathrm{I}=0.5 \mathrm{hy}_{1}+0.5 \mathrm{hy}_{2} \quad \text { Error } \approx \mathrm{O}\left(\mathrm{h}^{2}\right)
$$

Corresponding Author: Pedro Americo Almeida Magalhaes Junior, Pontificia Universidad Catolica de Minas Gerais, Av. Dom Jose Gaspar 500, 30535-610, Belo Horizonte, Minas Gerais, Brazil Tel: +55 31 3221-6813 
Simpson's $1 / 3$ rule is obtained by fitting a seconddegree polynomial to three equally spaced discrete points. The upper limit of integration is $\mathrm{x}_{3}$, then:

$$
\begin{aligned}
\mathrm{I} & =\frac{\mathrm{h}}{3}\left(\mathrm{y}_{1}+4 \mathrm{y}_{2}+\mathrm{y}_{3}\right) \quad \text { or } \quad \text { Error } \approx \mathrm{O}\left(\mathrm{h}^{4}\right) \\
\mathrm{I} & =0.33333333333333333333333333333333333333333 \mathrm{hy}_{1} \\
& +1.33333333333333333333333333333333333333333 \mathrm{hy}_{2} \\
& +0.33333333333333333333333333333333333333333 \mathrm{hy}_{3}
\end{aligned}
$$

Simpson's $3 / 8$ rule is obtained by fitting a thirddegree polynomial to four equally spaced discrete points. The upper limit of integration is $\mathrm{x}_{4}$, then:

$$
\begin{aligned}
& I=\frac{3 h}{8}\left(y_{1}+3 y_{2}+3 y_{3}+y_{4}\right) \text { or } \quad \text { Error } \approx O\left(h^{4}\right) \\
& I=0.375 h_{1}+1.125 h_{2}+1.125 h_{3}+0.375 h_{4}
\end{aligned}
$$

Boole's rule is obtained by fitting a fourth-degree polynomial to five equally spaced discrete points. The upper limit of integration is $\mathrm{x}_{5}$, then:

$$
\begin{aligned}
\mathrm{I} & =\frac{2 \mathrm{~h}}{45}\left(7 \mathrm{y}_{1}+32 \mathrm{y}_{2}+12 \mathrm{y}_{3}+32 \mathrm{y}_{4}+7 \mathrm{y}_{5}\right) \text { or Error } \approx \mathrm{O}\left(\mathrm{h}^{6}\right) \\
\mathrm{I} & =0.3111111111111111111111111111111111111111 \mathrm{hy}_{1} \\
& +1.422222222222222222222222222222222222222 \mathrm{hy}_{2} \\
& +0.5333333333333333333333333333333333333333 \mathrm{hy}_{3} \\
& +1.422222222222222222222222222222222222222 \mathrm{hy}_{4} \\
& +0.3111111111111111111111111111111111111111 \mathrm{hy}_{5}
\end{aligned}
$$

Generally, have the closed formulas, where $\mathrm{N}$ is number of points, ci are integer coefficients and cr are real coefficients:

$$
\begin{aligned}
& \mathrm{I}=\frac{\mathrm{num}}{\mathrm{den}} \mathrm{h}\left(\mathrm{ci}_{1} \mathrm{y}_{1}+\mathrm{ci}_{2} \mathrm{y}_{2}+\mathrm{ci}_{3} \mathrm{y}_{3}+\mathrm{ci}_{4} \mathrm{y}_{4}+\ldots\right. \\
& \left.+\mathrm{ci}_{\mathrm{N}-2} \mathrm{y}_{\mathrm{N}-2}+\mathrm{ci}_{\mathrm{N}-1} \mathrm{y}_{\mathrm{N}-1}+\mathrm{ci}_{\mathrm{N}} \mathrm{y}_{\mathrm{N}}\right) \\
& \text { or } \\
& \mathrm{I}=\mathrm{h}\left(\begin{array}{l}
\mathrm{cr}_{1} \mathrm{y}_{1}+\mathrm{cr}_{2} \mathrm{y}_{2}+\mathrm{cr}_{3} \mathrm{y}_{3}+\ldots \\
+\mathrm{cr}_{\mathrm{N}-2} \mathrm{y}_{\mathrm{N}-2}+\mathrm{cr}_{\mathrm{N}-1} \mathrm{y}_{\mathrm{N}-1}+\mathrm{cr}_{\mathrm{N}} \mathrm{y}_{\mathrm{N}}
\end{array}\right)
\end{aligned}
$$

\section{RESULTS AND DISCUSSION}

The Table 1 and 2 shows of coefficients for higher order closed integration formulas. Figure 1 shows the generation of rules in Maple 12.0®.

Newton-cotes open integration equation: In the open integration formulas, the first $\left(\mathrm{y}_{1}\right)$ and last $\left(\mathrm{y}_{\mathrm{N}}\right)$ point does not appear in equation. The midpoints rule for a double interval is obtained by fitting a zero-degree polynomial to three discrete points (Espelid, 2003). The upper limit of integration is $\mathrm{x}_{3}=\mathrm{x}_{1}+2 \mathrm{~h}$, then the integral (I) have the formula:

$$
\mathrm{I}=2 \mathrm{~h}\left(\mathrm{y}_{2}\right) \quad \text { or } \quad \mathrm{I}=2 \mathrm{hy}_{2} \quad \text { Error } \approx \mathrm{O}\left(\mathrm{h}^{2}\right)
$$

For three intervals, the rule is obtained by fitting a first-degree polynomial to four discrete points. The upper limit of integration is $\mathrm{x}_{4}=\mathrm{x}_{1}+3 \mathrm{~h}$, then the integral (I) have the formula:

$\mathrm{I}=\frac{3 \mathrm{~h}}{2}\left(\mathrm{y}_{2}+\mathrm{y}_{3}\right) \quad$ or $\quad \mathrm{I}=1.5 \mathrm{hy}_{2}+1.5 \mathrm{hy}_{3} \quad$ Error $\approx \mathrm{O}\left(\mathrm{h}^{2}\right)$

For $\mathrm{N}=5$, the rule is obtained by fitting a seconddegree polynomial to four equally spaced discrete points. The upper limit of integration is $\mathrm{x}_{5}$, then:

$$
\begin{aligned}
& I=\frac{4 h}{3}\left(2 y_{2}-y_{3}+2 y_{4}\right) \quad \text { or } \quad \text { Error } \approx O\left(h^{4}\right) \\
& I=2.6666666666666666666666666666666666666667 h y_{2} \\
& -1.333333333333333333333333333333333333333 \mathrm{hy}_{3} \\
& +2.666666666666666666666666666666666666667 \mathrm{hy}_{4}
\end{aligned}
$$

For $\mathrm{N}=6$, the rule is obtained by fitting a thirddegree polynomial to five equally spaced discrete points. The upper limit of integration is $\mathrm{x}_{6}$, then:

$$
\begin{aligned}
\mathrm{I} & =\frac{5 \mathrm{~h}}{24}\left(11 \mathrm{y}_{2}+\mathrm{y}_{3}+\mathrm{y}_{4}+11 \mathrm{y}_{5}\right) \quad \text { or } \quad \text { Error } \approx \mathrm{O}\left(\mathrm{h}^{4}\right) \\
\mathrm{I} & =2.2916666666666666666666666666666666666667 \mathrm{hy} \\
& +0.20833333333333333333333333333333333333333 \mathrm{hy}_{3} \\
& +0.2083333333333333333333333333333333333333 \mathrm{hy}_{4} \\
& +2.291666666666666666666666666666666666667 \mathrm{hy}_{5}
\end{aligned}
$$

Generally, have the open formulas, where $\mathrm{N}$ is number of points, ci are integer coefficients and cr are real coefficients:

$$
\begin{aligned}
& \mathrm{I}=\frac{\text { num }}{\operatorname{den}} \mathrm{h}\left(\mathrm{ci}_{2} \mathrm{y}_{2}+\mathrm{ci}_{3} \mathrm{y}_{3}+\mathrm{ci}_{4} \mathrm{y}_{4}+\ldots+\mathrm{ci}_{\mathrm{N}-2} \mathrm{y}_{\mathrm{N}-2}+\mathrm{ci}_{\mathrm{N}-1} \mathrm{y}_{\mathrm{N}-1}\right) \\
& \text { or } \quad \mathrm{I}=\mathrm{h}\left(\mathrm{cr}_{2} \mathrm{y}_{2}+\mathrm{cr}_{3} \mathrm{y}_{3}+\ldots+\mathrm{cr}_{\mathrm{N}-2} \mathrm{y}_{\mathrm{N}-2}+\mathrm{cr}_{\mathrm{N}-1} \mathrm{y}_{\mathrm{N}-1}\right)
\end{aligned}
$$

$$
\begin{aligned}
& \text { restart: } \\
& \text { for } \mathrm{N} \text { from } 2 \text { by } 1 \text { to } 105 \text { do } \\
& \text { assign }(\mathrm{x}, \operatorname{array}(1 . \mathrm{N})) \text { ) assign }(\mathrm{y}, \operatorname{array}(1 . \mathrm{N})) \text { : } \\
& \text { for } \mathrm{j} \text { from } 1 \text { by } 1 \text { to } \mathrm{N} \text { do } \mathrm{x}[\mathrm{j}]:=0+(\mathrm{j}-1)^{*} 1: \text { od: } \\
& \mathrm{p}:=\operatorname{sort}(\text { factor }(\operatorname{int}(\operatorname{interp}(\mathrm{x}, \mathrm{y}, \mathrm{xx}), \mathrm{xx}=\mathrm{x}[1] . \mathrm{x}[\mathrm{N}]))) \text {; } \\
& \mathrm{p} 1:=\operatorname{sort}\left(\operatorname{simplify}\left(\mathrm{p}^{*} \mathrm{~h}\right)\right) ; \\
& \mathrm{p} 2:=\operatorname{evalf}\left(\operatorname{sort}\left(\operatorname{expand}\left(\mathrm{p}^{*} \mathrm{~h}\right)\right), 40\right) \text {; } \\
& \text { for j from } 1 \text { by } 1 \text { to } \mathrm{N} \text { do print }(\operatorname{coeff}(\mathrm{p} 2, \mathrm{y}[\mathrm{j}])) \text {; od: } \\
& \text { od; }
\end{aligned}
$$

Fig. 1: Verification Newton-cotes closed integration formulas with maple $12.0 \AA$. (number of points $\mathrm{N}=2 . .105)$ 
J. Math. \& Stat., 6 (2): 193-204, 2010

Table 1: Newton-cotes closed integration formulas with points $=2.14$

\begin{tabular}{|c|c|c|}
\hline Rule & Integer coefficient & Real coefficient \\
\hline Trapezoidal rule & num $=1$ & \\
\hline Points $(\mathrm{N})=2$ & $\operatorname{den}=2$ & \\
\hline Segments $(\mathrm{N}-1)=1$ & $\mathrm{ci}_{1}=\mathrm{ci}_{2}=1$ & $\mathrm{cr}_{1}=\mathrm{cr}_{2}=+0.5000000000000000000000000000000000000000$ \\
\hline \multicolumn{3}{|r|}{ W } \\
\hline Simpson's $1 / 3$ rule & num $=1$ & \\
\hline Points $(\mathrm{N})=3$ & $\operatorname{den}=3$ & \\
\hline Segments $(\mathrm{N}-1)=2$ & $\mathrm{ci}_{1}=\mathrm{ci}_{3}=1$ & $\mathrm{cr}_{1}=\mathrm{cr}_{3}=+0.3333333333333333333333333333333333333333$ \\
\hline Error»O $\left(h^{4}\right)$ & $\mathrm{ci}_{2}=4$ & $\mathrm{cr}_{2}=+1.33333333333333333333333333333333333333333$ \\
\hline Simpson's $3 / 8$ rule & num $=3$ & \\
\hline Points $(N)=4$ & $\operatorname{den}=8$ & \\
\hline Segments $(N-1)=3$ & $\mathrm{ci}_{1}=\mathrm{ci}_{4}=1$ & $\mathrm{cr}_{1}=\mathrm{cr}_{4}=+0.3750000000000000000000000000000000000000$ \\
\hline Error» $\mathrm{O}\left(\mathrm{h}^{4}\right)$ & $\mathrm{ci}_{2}=\mathrm{ci}_{3}=3$ & $\mathrm{cr}_{2}=\mathrm{cr}_{3}=+1.1250000000000000000000000000000000000000$ \\
\hline Boole's rule & num $=2$ & \\
\hline Points $(\mathrm{N})=5$ & $\operatorname{den}=45$ & \\
\hline Segments $(N-1)=4$ & $\mathrm{ci}_{1}=\mathrm{ci}_{5}=7$ & $\mathrm{cr}_{1}=\mathrm{cr}_{5}=+0.311111111111111111111111111111111111111$ \\
\hline \multirow[t]{2}{*}{ Error» $\mathrm{O}\left(\mathrm{h}^{6}\right)$} & $\mathrm{ci}_{2}=\mathrm{ci}_{4}=32$ & $\mathrm{cr}_{2}=\mathrm{cr}_{4}=+1.4222222222222222222222222222222222222222$ \\
\hline & $\begin{array}{l}\mathrm{ci}_{3}=12 \\
\text { num }=5\end{array}$ & $\mathrm{cr}_{3}=+0.5333333333333333333333333333333333333333$ \\
\hline Points $(N)=6$ & den $=288$ & \\
\hline Segments $(\mathrm{N}-1)=5$ & $\mathrm{ci}_{1}=\mathrm{ci}_{6}=19$ & $\mathrm{cr}_{1}=\mathrm{cr}_{6}=+0.3298611111111111111111111111111111111111$ \\
\hline \multirow[t]{2}{*}{ Error»O $\left(h^{6}\right)$} & $\mathrm{ci}_{2}=\mathrm{ci}_{5}=75$ & $\mathrm{cr}_{2}=\mathrm{cr}_{5}=+1.3020833333333333333333333333333333333333$ \\
\hline & $\mathrm{ci}_{3}=\mathrm{ci}_{4}=50$ & $\mathrm{cr}_{3}=\mathrm{cr}_{4}=+0.8680555555555555555555555555555555555556$ \\
\hline Points $(N)=7$ & $\begin{array}{l}\text { num }=1 \\
\text { den }=140\end{array}$ & \\
\hline Segments $(N-1)=6$ & $\mathrm{ci}_{1}=\mathrm{ci}_{7}=41$ & $\mathrm{cr}_{1}=\mathrm{cr}_{7}=+0.2928571428571428571428571428571428571429$ \\
\hline \multirow[t]{4}{*}{ Error»O $\left(h^{8}\right)$} & $\mathrm{ci}_{2}=\mathrm{ci}_{6}=216$ & $\mathrm{cr}_{2}=\mathrm{cr}_{6}=+1.5428571428571428571428571428571428571429$ \\
\hline & $\mathrm{ci}_{3}=\mathrm{ci}_{5}=27$ & $\mathrm{cr}_{3}=\mathrm{cr}_{5}=+0.1928571428571428571428571428571428571429$ \\
\hline & $\mathrm{ci}_{4}=272$ & $\mathrm{cr}_{4}=+1.9428571428571428571428571428571428571429$ \\
\hline & num $=7$ & \\
\hline Points $(N)=8$ & $\operatorname{den}=17280$ & \\
\hline Segments $(N-1)=7$ & $\mathrm{ci}_{1}=\mathrm{ci}_{8}=751$ & $\mathrm{cr}_{1}=\mathrm{cr}_{8}=+0.3042245370370370370370370370370370370370$ \\
\hline \multirow[t]{4}{*}{ Error» $\mathrm{O}\left(\mathrm{h}^{8}\right)$} & $\mathrm{ci}_{2}=\mathrm{ci}_{7}=3577$ & $\mathrm{cr}_{2}=\mathrm{cr}_{7}=+1.4490162037037037037037037037037037037037$ \\
\hline & $\mathrm{ci}_{3}=\mathrm{ci}_{6}=1323$ & $\mathrm{cr}_{3}=\mathrm{cr}_{6}=+0.5359375000000000000000000000000000000000$ \\
\hline & $\mathrm{ci}_{4}=\mathrm{ci}_{5}=2989$ & $\mathrm{cr}_{4}=\mathrm{cr}_{5}=+1.2108217592592592592592592592592592592593$ \\
\hline & num $=4$ & \\
\hline Points $(\mathrm{N})=9$ & $\operatorname{den}=14175$ & \\
\hline Segments $(\mathrm{N}-1)=8$ & $\mathrm{ci}_{1}=\mathrm{ci}_{9}=989$ & $\mathrm{cr}_{1}=\mathrm{cr}_{9}=+0.2790828924162257495590828924162257495591$ \\
\hline \multirow[t]{5}{*}{ Error»O $\left(h^{10}\right)$} & $\mathrm{ci}_{2}=\mathrm{ci}_{8}=5888$ & $\mathrm{cr}_{2}=\mathrm{cr}_{8}=+1.6615167548500881834215167548500881834215$ \\
\hline & $\mathrm{ci}_{3}=\mathrm{ci}_{7}=-928$ & $\mathrm{cr}_{3}=\mathrm{cr}_{7}=-0.2618694885361552028218694885361552028219$ \\
\hline & $\mathrm{ci}_{4}=\mathrm{ci}_{6}=10496$ & $\mathrm{cr}_{4}=\mathrm{cr}_{6}=+2.9618342151675485008818342151675485008818$ \\
\hline & $\mathrm{ci}_{5}=-4540$ & $\mathrm{cr}_{5}=-1.2811287477954144620811287477954144620811$ \\
\hline & num $=9$ & \\
\hline Points $(\mathrm{N})=10$ & $\operatorname{den}=89600$ & \\
\hline Segments $(N-1)=9$ & $\mathrm{ci}_{1}=\mathrm{ci}_{10}=2857$ & $\mathrm{cr}_{1}=\mathrm{cr}_{10}=+0.2869754464285714285714285714285714285714$ \\
\hline \multirow[t]{4}{*}{ Error»O $\left(h^{10}\right)$} & $\mathrm{ci}_{2}=\mathrm{ci}_{9}=15741$ & $\mathrm{cr}_{2}=\mathrm{cr}_{9}=+1.5811272321428571428571428571428571428571$ \\
\hline & $\mathrm{ci}_{3}=\mathrm{ci}_{8}=1080$ & $\mathrm{cr}_{3}=\mathrm{cr}_{8}=+0.1084821428571428571428571428571428571429$ \\
\hline & $\mathrm{ci}_{4}=\mathrm{ci}_{7}=19344$ & $\mathrm{cr}_{4}=\mathrm{cr}_{7}=+1.9430357142857142857142857142857142857143$ \\
\hline & $\begin{array}{l}\mathrm{ci}_{5}=\mathrm{ci}_{6}=5778 \\
\text { num }=5\end{array}$ & $\mathrm{cr}_{5}=\mathrm{cr}_{6}=+0.5803794642857142857142857142857142857143$ \\
\hline Points $(\mathrm{N})=11$ & den $=299376$ & \\
\hline Segments $(N-1)=10$ & $\mathrm{ci}_{1}=\mathrm{ci}_{11}=16067$ & $\mathrm{cr}_{1}=\mathrm{cr}_{11}=+0.2683414836192613970391748169525947303725$ \\
\hline \multirow[t]{6}{*}{ Error» $\mathrm{O}\left(\mathrm{h}^{12}\right)$} & $\mathrm{ci}_{2}=\mathrm{ci}_{10}=106300$ & $\mathrm{cr}_{2}=\mathrm{cr}_{10}=+1.775359414248303137192026080914969803859$ \\
\hline & $\mathrm{ci}_{3}=\mathrm{ci}_{9}=-48525$ & $\mathrm{cr}_{3}=\mathrm{cr}_{9}=-0.8104357062690396023729357062690396023729$ \\
\hline & $\mathrm{ci}_{4}=\mathrm{ci}_{8}=272400$ & $\mathrm{cr}_{4}=\mathrm{cr}_{8}=+4.549462882796216129549462882796216129549$ \\
\hline & $\mathrm{ci}_{5}=\mathrm{ci}_{7}=-260550$ & $\mathrm{cr}_{5}=\mathrm{cr}_{7}=-4.351551226551226551226551226551226551227$ \\
\hline & $\mathrm{ci}_{6}=427368$ & $\mathrm{cr}_{6}=+7.137646304312970979637646304312970979638$ \\
\hline & num $=11$ & \\
\hline Points $(\mathrm{N})=12$ & $\mathrm{den}=87091200$ & \\
\hline Segments $(N-1)=11$ & $\mathrm{ci}_{1}=\mathrm{ci}_{12}=2171465$ & $\mathrm{cr}_{1}=\mathrm{cr}_{12}=+0.2742655400315990593768371546149323927102$ \\
\hline \multirow[t]{5}{*}{ Error» $\mathrm{O}\left(\mathrm{h}^{12}\right)$} & $\mathrm{ci}_{2}=\mathrm{ci}_{11}=13486539$ & $\mathrm{cr}_{2}=\mathrm{cr}_{11}=+1.703408943727954144620811287477954144621$ \\
\hline & $\mathrm{ci}_{3}=\mathrm{ci}_{10}=-3237113$ & $\mathrm{cr}_{3}=\mathrm{cr}_{10}=-0.4088615497317754262198706643151087595532$ \\
\hline & $\mathrm{ci}_{4}=\mathrm{ci}_{9}=25226685$ & $\mathrm{cr}_{4}=\mathrm{cr}_{9}=+3.186240802744708994708994708994708994709$ \\
\hline & $\mathrm{ci}_{5}=\mathrm{ci}_{8}=-9595542$ & $\mathrm{cr}_{5}=\mathrm{cr}_{8}=-1.211958980930335097001763668430335097002$ \\
\hline & $\mathrm{ci}_{6}=\mathrm{ci}_{7}=15493566$ & $\mathrm{cr}_{6}=\mathrm{cr}_{7}=+1.956905244157848324514991181657848324515$ \\
\hline
\end{tabular}


Table 1: Continued

\begin{tabular}{|c|c|c|}
\hline Points $(\mathrm{N})=13$ & $\begin{array}{l}\text { num }=1 \\
\text { den }=5255250\end{array}$ & \\
\hline Segments $(\mathrm{N}-1)=12$ & $\mathrm{ci}_{1}=\mathrm{ci}_{13}=1364651$ & $\mathrm{cr}_{1}=\mathrm{cr}_{13}=+0.2596738499595642452785309928167071024214$ \\
\hline \multirow[t]{6}{*}{ Error»O $\left(\mathrm{h}^{14}\right)$} & $\mathrm{ci}_{2}=\mathrm{ci}_{12}=9903168$ & $\mathrm{cr}_{2}=\mathrm{cr}_{12}=+1.884433281004709576138147566718995290424$ \\
\hline & $\mathrm{ci}_{3}=\mathrm{ci}_{11}=-7587864$ & $\mathrm{cr}_{3}=\mathrm{cr}_{11}=-1.443863565006422149279292136434993577851$ \\
\hline & $\mathrm{ci}_{4}=\mathrm{ci}_{10}=35725120$ & $\mathrm{cr}_{4}=\mathrm{cr}_{10}=+6.797986775129632272489415346558203701061$ \\
\hline & $\mathrm{ci}_{5}=\mathrm{ci}_{9}=-51491295$ & $\mathrm{cr}_{5}=\mathrm{cr}_{9}=-9.798067646639075210503781932353360924789$ \\
\hline & $\mathrm{ci}_{6}=\mathrm{ci}_{8}=87516288$ & $\mathrm{cr}_{6}=\mathrm{cr}_{8}=+16.65311602683031254459825888397316968746$ \\
\hline & $\begin{array}{l}\mathrm{ci}_{7}=-87797136 \\
\text { num }=13\end{array}$ & $\mathrm{cr}_{7}=-16.70655744255744255744255744255744255744$ \\
\hline Points $(N)=14$ & $\operatorname{den}=402361344000$ & \\
\hline Segments $(N-1)=13$ & $\mathrm{ci}_{1}=\mathrm{ci}_{14}=8181904909$ & $\mathrm{cr}_{1}=\mathrm{cr}_{14}=+0.2643513483666065097943404821711700018578$ \\
\hline \multirow[t]{6}{*}{ Error» $\mathrm{O}\left(\mathrm{h}^{14}\right)$} & $\mathrm{ci}_{2}=\mathrm{ci}_{13}=56280729661$ & $\mathrm{cr}_{2}=\mathrm{cr}_{13}=+1.818389108455209852365937022021678106334$ \\
\hline & $\mathrm{ci}_{3}=\mathrm{ci}_{12}=-31268252574$ & $\mathrm{cr}_{3}=\mathrm{cr}_{12}=-1.010254313749384433908243432052955862480$ \\
\hline & $\mathrm{ci}_{4}=\mathrm{ci}_{11}=156074417954$ & $\mathrm{cr}_{4}=\mathrm{cr}_{11}=+5.042650005170476813995332513851032369551$ \\
\hline & $\mathrm{ci}_{5}=\mathrm{ci}_{10}=-151659573325$ & $\mathrm{cr}_{5}=\mathrm{cr}_{10}=-4.900009612317529190875751722312568873415$ \\
\hline & $\mathrm{ci}_{6}=\mathrm{ci}_{9}=206683437987$ & $\mathrm{cr}_{6}=\mathrm{cr}_{9}=+6.677790334230019869900822281774662727044$ \\
\hline & $\mathrm{ci}_{7}=\mathrm{ci}_{8}=-43111992612$ & $\mathrm{cr}_{7}=\mathrm{cr}_{8}=-1.392916870155399421272437145453018468891$ \\
\hline
\end{tabular}

Table 2: Newton-cotes closed integration formulas with points $=15.21$

Rule
Points $(\mathrm{N})=15$
Segments $(\mathrm{N}-1)=14$
Error» $\mathrm{O}\left(\mathrm{h}^{16}\right)$

Points $(\mathrm{N})=16$

Segments $(\mathrm{N}-1)=15$ Error» $\mathrm{O}\left(\mathrm{h}^{16}\right)$

Points $(\mathrm{N})=17$ Segments $(\mathrm{N}-1)=16$ Error»O $\left(\mathrm{h}^{18}\right)$

Points $(\mathrm{N})=18$

Segments $(\mathrm{N}-1)=17$ Error»O $\left(h^{18}\right)$

Points $(\mathrm{N})=19$
Integer coefficient num $=7$

den $=2501928000$

$\mathrm{ci}_{1}=\mathrm{ci}_{15}=90241897$

$\mathrm{ci}_{2}=\mathrm{ci}_{14}=710986864$

$\mathrm{ci}_{3}=\mathrm{ci}_{13}=-770720657$

$\mathrm{ci}_{4}=\mathrm{ci}_{12}=3501442784$

$\mathrm{ci}_{5}=\mathrm{ci}_{11}=-6625093363$

$\mathrm{ci}_{6}=\mathrm{ci}_{10}=12630121616$

$\mathrm{ci}_{7}=\mathrm{ci}_{9}=-16802270373$

$\mathrm{ci}_{8}=19534438464$

num $=5$

$\operatorname{den}=688816128$

$\mathrm{ci}_{1}=\mathrm{ci}_{16}=35310023$

$\mathrm{ci}_{2}=\mathrm{ci}_{15}=265553865$

$\mathrm{ci}_{3}=\mathrm{ci}_{14}=-232936065$

$\mathrm{ci}_{4}=\mathrm{ci}_{13}=1047777585$

$\mathrm{ci}_{5}=\mathrm{ci}_{12}=-1562840685$

$\mathrm{ci}_{6}=\mathrm{ci}_{11}=2461884669$

$\mathrm{ci}_{7}=\mathrm{ci}_{10}=-2000332805$

$\mathrm{ci}_{8}=\mathrm{ci}_{9}=1018807605$

num $=8$

den $=488462349375$

$\mathrm{ci}_{1}=\mathrm{ci}_{17}=15043611773$

$\mathrm{ci}_{2}=\mathrm{ci}_{16}=127626606592$

$\mathrm{ci}_{3}=\mathrm{ci}_{15}=-179731134720$

$\mathrm{ci}_{4}=\mathrm{ci}_{14}=832211855360$

$\mathrm{ci}_{5}=\mathrm{ci}_{13}=-1929498607520$

$\mathrm{ci}_{6}=\mathrm{ci}_{12}=4177588893696$

$\mathrm{ci}_{7}=\mathrm{ci}_{11}=-6806534407936$

$\mathrm{ci}_{8}=\mathrm{ci}_{10}=9368875018240$

$\mathrm{ci}_{9}=-10234238972220$

num $=17$

den $=3766102179840000$

$\mathrm{ci}_{1}=\mathrm{ci}_{18}=55294720874657$

$\mathrm{ci}_{2}=\mathrm{ci}_{17}=450185515446285$

$\mathrm{ci}_{3}=\mathrm{ci}_{16}=-542023437008852$

$\mathrm{ci}_{4}=\mathrm{ci}_{15}=2428636525764260$

$\mathrm{ci}_{5}=\mathrm{ci}_{14}=-4768916800123440$

$\mathrm{ci}_{6}=\mathrm{ci}_{13}=8855416648684984$

$\mathrm{ci}_{7}=\mathrm{ci}_{12}=-10905371859796660$

$\mathrm{ci}_{8}=\mathrm{ci}_{11}=10069615750132836$

$\mathrm{ci}_{9}=\mathrm{ci}_{10}=-3759785974054070$

num $=3$

den $=2534852320000$
Real coefficient

$\mathrm{cr}_{1}=\mathrm{cr}_{15}=+0.2524825970211772680908483377619180088316$ $\mathrm{cr}_{2}=\mathrm{cr}_{14}=+1.989229125698261500730636533105668908138$ $\mathrm{cr}_{3}=\mathrm{cr}_{13}=-2.156354858732945152698239117992204411957$ $\mathrm{cr}_{4}=\mathrm{cr}_{12}=+9.796484746163758509437521783200795546475$ $\mathrm{cr}_{5}=\mathrm{cr}_{11}=-18.53596647905135559456547110868098522420$ $\mathrm{cr}_{6}=\mathrm{cr}_{10}=+35.33708856210090777992012559913794481696$ $\mathrm{cr}_{7}=\mathrm{cr}_{9}=-47.01010285307970493155678340863526048711$ $\mathrm{cr}_{8}=+54.65427831975980124128272276420424568573$

$\mathrm{cr}_{1}=\mathrm{cr}_{16}=+0.2563094965743891525141525141525141525142$ $\mathrm{cr}_{2}=\mathrm{cr}_{15}=+1.927610680161077761524190095618667047238$ $\mathrm{cr}_{3}=\mathrm{cr}_{14}=-1.690843575892578403739118024832310546596$ $\mathrm{cr}_{4}=\mathrm{cr}_{13}=+7.605640623153353343085485942628799771657$ $\mathrm{cr}_{5}=\mathrm{cr}_{12}=-11.34439672266210352147852147852147852148$ $\mathrm{cr}_{6}=\mathrm{cr}_{11}=+17.87040524260198506850292564578278863993$ $\mathrm{cr}_{7}=\mathrm{cr}_{10}=-14.52007817244372071380999952428523857095$ $\mathrm{cr}_{8}=\mathrm{cr}_{9}=+7.395352428507597313400884829456258027687$

$\mathrm{cr}_{1}=\mathrm{cr}_{17}=+0.2463831538663920999571309077827734673189$ $\mathrm{cr}_{2}=\mathrm{cr}_{16}=+2.090259063042242486859839973925114154045$ $\mathrm{cr}_{3}=\mathrm{cr}_{15}=-2.943623146389408449788157226688194630927$ $\mathrm{cr}_{4}=\mathrm{cr}_{14}=+13.62990382247206952397670660284347734636$ $\mathrm{cr}_{5}=\mathrm{cr}_{13}=-31.60118457422714434160988091202152135167$ $\mathrm{cr}_{6}=\mathrm{cr}_{12}=+68.42023994752236271066025189896960827555$ $\mathrm{cr}_{7}=\mathrm{cr}_{11}=-111.4769138975830403018562232865238017916$ $\mathrm{cr}_{8}=\mathrm{cr}_{10}=+153.4427376886298627834748455836397185776$ $\mathrm{cr}_{9}=-167.6156041146666730233490270838543480934$

$\mathrm{cr}_{1}=\mathrm{cr}_{18}=+0.2495976502977156674085870147010652595603$ $\mathrm{cr}_{2}=\mathrm{cr}_{17}=+2.032115273864391922530976763780996585229$ $\mathrm{cr}_{3}=\mathrm{cr}_{16}=-2.446667134650592709501072228879401131018$ $\mathrm{cr}_{4}=\mathrm{cr}_{15}=+10.96274582219286979928698035693920467583$ $\mathrm{cr}_{5}=\mathrm{cr}_{14}=-21.52665587144073317188396553475918555284$ $\mathrm{cr}_{6}=\mathrm{cr}_{13}=+39.97291518894487202421873203766207881434$ $\mathrm{cr}_{7}=\mathrm{cr}_{12}=-49.22631218264487713639866785075485839742$ $\mathrm{cr}_{8}=\mathrm{cr}_{11}=+45.45375021118805970203126287782372438457$ $\mathrm{cr}_{9}=\mathrm{cr}_{10}=-16.97148895775170609769283343651362463826$ 
Table 2: Continued

Segments (N-1) $=18$ Error»O $\left(\mathrm{h}^{20}\right)$

Points $(\mathrm{N})=20$ Segments $(\mathrm{N}-1)=19$ Error»O $\left(h^{20}\right)$

Points $(\mathrm{N})=21$ Segments $(\mathrm{N}-1)=20$ Error»O $\left(h^{22}\right)$

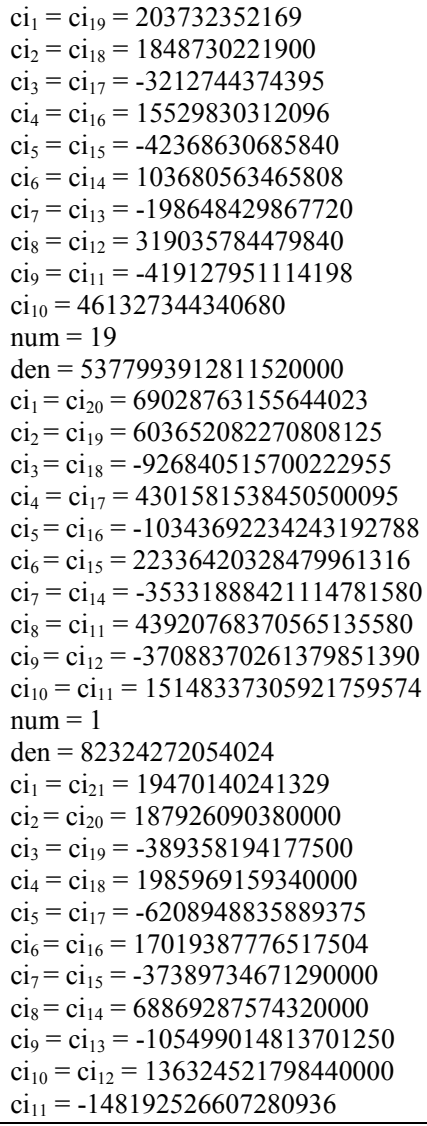

$\mathrm{cr}_{1}=\mathrm{cr}_{19}=+0.2411174219833840260958476665812231617501$ $\mathrm{cr}_{2}=\mathrm{cr}_{18}=+2.187973879953685033611741136856446138054$ $\mathrm{cr}_{3}=\mathrm{cr}_{17}=-3.802285855921184394679055701359359664787$ $\mathrm{cr}_{4}=\mathrm{cr}_{16}=+18.37956813842630485076937342053914998882$ $\mathrm{cr}_{5}=\mathrm{cr}_{15}=-50.14331251357475531355609702738027752244$ $\mathrm{cr}_{6}=\mathrm{cr}_{14}=+122.7060400889247859614953821057315086506$ $\mathrm{cr}_{7}=\mathrm{cr}_{13}=-235.1005953684749571525334462088110916063$ $\mathrm{cr}_{8}=\mathrm{cr}_{12}=+377.5791378014163760041058328794475884891$ $\mathrm{cr}_{9}=\mathrm{cr}_{11}=-496.0383070137174697419848111703801348080$ $\mathrm{cr}_{10}=+545.9813268419676614533504657975498943465$

$\mathrm{cr}_{1}=\mathrm{cr}_{20}=+0.2438728122828207419466199855927763551718$ $\mathrm{cr}_{2}=\mathrm{cr}_{19}=+2.132652016548929222835872365540199752074$ $\mathrm{cr}_{3}=\mathrm{cr}_{18}=-3.274449559407934643744028261724229000028$ $\mathrm{cr}_{4}=\mathrm{cr}_{17}=+15.19712564862917055652934244339942421644$ $\mathrm{cr}_{5}=\mathrm{cr}_{16}=-36.54339436540532989294062010185496617106$ $\mathrm{cr}_{6}=\mathrm{cr}_{15}=+78.91269367749333291376931749747542731403$ $\mathrm{cr}_{7}=\mathrm{cr}_{14}=-124.8245890353256315709366953012559403264$ $\mathrm{cr}_{8}=\mathrm{cr}_{13}=+155.1683792450554438067301044281960105079$ $\mathrm{cr}_{9}=\mathrm{cr}_{12}=-131.0300915900113864767933860848804604084$ $\mathrm{cr}_{10}=\mathrm{cr}_{11}=+53.51780115014058534260347302951175776026$

$\mathrm{cr}_{1}=\mathrm{cr}_{21}=+0.2365054649806320638934570035459272429452$ $\mathrm{cr}_{2}=\mathrm{cr}_{20}=+2.282754352892139499749904325488735190028$ $\mathrm{cr}_{3}=\mathrm{cr}_{19}=-4.729567410228539284620863621941806709062$ $\mathrm{cr}_{4}=\mathrm{cr}_{18}=+24.12373786963751328807016941644254281952$ $\mathrm{cr}_{5}=\mathrm{cr}_{17}=-75.42063453430660935475529909597864068132$ $\mathrm{cr}_{6}=\mathrm{cr}_{16}=+206.7359643987960228706236680773493746266$ $\mathrm{cr}_{7}=\mathrm{cr}_{15}=-454.1763168795902459592599440453836115659$ $\mathrm{cr}_{8}=\mathrm{cr}_{14}=+836.5611484438710920695212236074943415938$ $\mathrm{cr}_{9}=\mathrm{cr}_{13}=-1281.505589803080093031109962977714705574$ $\mathrm{cr}_{10}=\mathrm{cr}_{12}=+1655.945669449457034417049362703788321739$ $\mathrm{cr}_{11}=-1800.107342704857893158323430786180957363$

Table 3: Newton-cotes open integration formulas with points $=3.16$

\begin{tabular}{|c|c|c|c|}
\hline Rule & Integer coefficient & Real coefficient & \\
\hline Midpoint rule & num $=2$ & & \\
\hline Points $(N)=3$ & den $=1$ & & \\
\hline Segments $(N-1)=2$ & $\mathrm{ci}_{2}=1$ & & $\mathrm{cr}_{2}=+2.00000000000000000000000000000000000000000$ \\
\hline \multicolumn{4}{|r|}{ 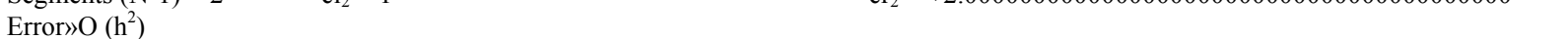 } \\
\hline Points $(\mathrm{N})=4$ & num $=3$ & & \\
\hline Segments $(N-1)=3$ & den $=2$ & & \\
\hline Error»O $\left(h^{2}\right)$ & $\mathrm{ci}_{2}=\mathrm{ci}_{3}=1$ & & $\mathrm{cr}_{2}=\mathrm{cr}_{3}=+1.5000000000000000000000000000000000000000$ \\
\hline Points $(\mathrm{N})=5$ & num $=4$ & $\operatorname{den}=3$ & \\
\hline Segments $(N-1)=4$ & $\mathrm{ci}_{2}=\mathrm{ci}_{4}=2$ & & $\mathrm{cr}_{2}=\mathrm{cr}_{4}=+2.66666666666666666666666666666666666666667$ \\
\hline Error»O $\left(h^{4}\right)$ & $\mathrm{ci}_{3}=-1$ & & $\mathrm{cr}_{3}=-1.3333333333333333333333333333333333333333$ \\
\hline Points $(N)=6$ & num $=5$ & $\operatorname{den}=24$ & \\
\hline Segments $(\mathrm{N}-1)=5$ & $\mathrm{ci}_{2}=\mathrm{ci}_{5}=11$ & & $\mathrm{cr}_{2}=\mathrm{cr}_{5}=+2.29166666666666666666666666666666666666667$ \\
\hline Error»O $\left(h^{4}\right)$ & $\mathrm{ci}_{3}=\mathrm{ci}_{4}=1$ & & $\mathrm{cr}_{3}=\mathrm{cr}_{4}=+0.2083333333333333333333333333333333333333$ \\
\hline Points $(N)=7$ & num $=3$ & $\operatorname{den}=10$ & \\
\hline Segments $(N-1)=6$ & $\mathrm{ci}_{2}=\mathrm{ci}_{6}=11$ & & $\mathrm{cr}_{2}=\mathrm{cr}_{6}=+3.3000000000000000000000000000000000000000$ \\
\hline \multirow[t]{2}{*}{ Error»O $\left(h^{6}\right)$} & $\mathrm{ci}_{2}=\mathrm{ci}_{5}=-14$ & & $\mathrm{cr}_{3}=\mathrm{cr}_{5}=-4.2000000000000000000000000000000000000000$ \\
\hline & $\mathrm{ci}_{4}=26$ & & $\mathrm{cr}_{4}=+7.8000000000000000000000000000000000000000$ \\
\hline Points $(\mathrm{N})=8$ & num $=7$ & $\operatorname{den}=1440$ & \\
\hline Segments $(N-1)=7$ & $\mathrm{ci}_{2}=\mathrm{ci}_{7}=611$ & & $\mathrm{cr}_{2}=\mathrm{cr}_{7}=+2.97013888888888888888888888888888888888889$ \\
\hline \multirow[t]{2}{*}{ Error»O $\left(h^{6}\right)$} & $\mathrm{ci}_{3}=\mathrm{ci}_{6}=-453$ & & $\mathrm{cr}_{3}=\mathrm{cr}_{6}=-2.20208333333333333333333333333333333333333$ \\
\hline & $\mathrm{ci}_{4}=\mathrm{ci}_{6}=562$ & & $\mathrm{cr}_{4}=\mathrm{cr}_{5}=+2.73194444444444444444444444444444444444444$ \\
\hline Points $(N)=9$ & num $=8$ & den $=945$ & \\
\hline Segments $(N-1)=8$ & $\mathrm{ci}_{2}=\mathrm{ci}_{8}=460$ & & $\mathrm{cr}_{2}=\mathrm{cr}_{8}=+3.894179894179894179894179894179894179894$ \\
\hline \multirow[t]{3}{*}{ Error»O $\left(\mathrm{h}^{8}\right)$} & $\mathrm{ci}_{3}=\mathrm{ci}_{7}=-954$ & & $\mathrm{cr}_{3}=\mathrm{cr}_{7}=-8.076190476190476190476190476190476190476$ \\
\hline & $\mathrm{ci}_{4}=\mathrm{ci}_{6}=2196$ & & $\mathrm{cr}_{4}=\mathrm{cr}_{6}=+18.59047619047619047619047619047619047619$ \\
\hline & $\mathrm{ci}_{5}=-2459$ & & $\mathrm{cr}_{5}=-20.81693121693121693121693121693121693122$ \\
\hline
\end{tabular}


J. Math. \& Stat., 6 (2): 193-204, 2010

Table 3: Continued

\begin{tabular}{|c|c|c|}
\hline Points $(\mathrm{N})=10$ & den $=4480$ & \\
\hline Segments $(\mathrm{N}-1)=9$ & $\mathrm{ci}_{2}=\mathrm{ci}_{9}=1787$ & $\mathrm{cr}_{2}=\mathrm{cr}_{9}=+3.589955357142857142857142857142857142857$ \\
\hline \multirow[t]{3}{*}{ Error» $\mathrm{O}\left(\mathrm{h}^{8}\right)$} & $\mathrm{ci}_{3}=\mathrm{ci}_{8}=-2803$ & $\mathrm{cr}_{3}=\mathrm{cr}_{8}=-5.631026785714285714285714285714285714286$ \\
\hline & $\mathrm{ci}_{4}=\mathrm{ci}_{7}=4967$ & $\mathrm{cr}_{4}=\mathrm{cr}_{7}=+9.978348214285714285714285714285714285714$ \\
\hline & $\begin{array}{l}\mathrm{ci}_{5}=\mathrm{ci}_{6}=-1711 \\
\text { num }=5\end{array}$ & $\mathrm{cr}_{5}=\mathrm{cr}_{6}=-3.437276785714285714285714285714285714286$ \\
\hline Points $(\mathrm{N})=11$ & $\mathrm{den}=4536$ & \\
\hline Segments $(\mathrm{N}-1)=10$ & $\mathrm{ci}_{2}=\mathrm{ci}_{10}=4045$ & $\mathrm{cr}_{2}=\mathrm{cr}_{10}=+4.458774250440917107583774250440917107584$ \\
\hline \multirow[t]{5}{*}{ Error»O $\left(h^{10}\right)$} & $\mathrm{ci}_{3}=\mathrm{ci}_{9}=-11690$ & $\mathrm{cr}_{3}=\mathrm{cr}_{9}=-12.88580246913580246913580246913580246914$ \\
\hline & $\mathrm{ci}_{4}=\mathrm{ci}_{8}=33340$ & $\mathrm{cr}_{4}=\mathrm{cr}_{8}=+36.75044091710758377425044091710758377425$ \\
\hline & $\mathrm{ci}_{5}=\mathrm{ci}_{7}=-55070$ & $\mathrm{cr}_{5}=\mathrm{cr}_{7}=-60.70326278659611992945326278659611992945$ \\
\hline & $\mathrm{ci}_{6}=67822$ & $\mathrm{cr}_{6}=+74.75970017636684303350970017636684303351$ \\
\hline & num $=11$ & \\
\hline Points $(\mathrm{N})=12$ & den $=7257600$ & \\
\hline Segments $(\mathrm{N}-1)=11$ & $\mathrm{ci}_{2}=\mathrm{ci}_{11}=2752477$ & $\mathrm{cr}_{2}=\mathrm{cr}_{11}=+4.171798804012345679012345679012345679012$ \\
\hline \multirow[t]{4}{*}{ Error»O $\left(h^{10}\right)$} & $\mathrm{ci}_{3}=\mathrm{ci}_{10}=-6603199$ & $\mathrm{cr}_{3}=\mathrm{cr}_{10}=-10.00815545083774250440917107583774250441$ \\
\hline & $\mathrm{ci}_{4}=\mathrm{ci}_{9}=15673880$ & $\mathrm{cr}_{4}=\mathrm{cr}_{9}=+23.75615630511463844797178130511463844797$ \\
\hline & $\mathrm{ci}_{5}=\mathrm{ci}_{8}=-17085616$ & $\mathrm{cr}_{5}=\mathrm{cr}_{8}=-25.89585758377425044091710758377425044092$ \\
\hline & $\begin{array}{l}\mathrm{ci}_{6}=\mathrm{ci}_{7}=8891258 \\
\text { num }=1\end{array}$ & $\mathrm{cr}_{6}=\mathrm{cr}_{7}=+13.47605792548500881834215167548500881834$ \\
\hline Points $(\mathrm{N})=13$ & den $=1925$ & \\
\hline Segments $(\mathrm{N}-1)=12$ & $\mathrm{ci}_{2}=\mathrm{ci}_{12}=9626$ & $\mathrm{cr}_{2}=\mathrm{cr}_{12}=+5.000519480519480519480519480519480519481$ \\
\hline \multirow[t]{5}{*}{ Error»O $\left(\mathrm{h}^{12}\right)$} & $\mathrm{ci}_{3}=\mathrm{ci}_{11}=-35771$ & $\mathrm{cr}_{3}=\mathrm{cr}_{11}=-18.58233766233766233766233766233766233766$ \\
\hline & $\mathrm{ci}_{4}=\mathrm{ci}_{10}=123058$ & $\mathrm{cr}_{4}=\mathrm{cr}_{10}=+63.92623376623376623376623376623376623377$ \\
\hline & $\mathrm{ci}_{5}=\mathrm{ci}_{9}=-266298$ & $\mathrm{cr}_{5}=\mathrm{cr}_{9}=-138.3366233766233766233766233766233766234$ \\
\hline & $\mathrm{ci}_{6}=\mathrm{ci}_{8}=427956$ & $\mathrm{cr}_{6}=\mathrm{cr}_{8}=+222.3148051948051948051948051948051948052$ \\
\hline & $\mathrm{ci}_{7}=-494042$ & $\mathrm{cr}_{7}=-256.6451948051948051948051948051948051948$ \\
\hline Points $(\mathrm{N})=14$ & den $=958003200$ & \\
\hline Segments $(\mathrm{N}-1)=13$ & $\mathrm{ci}_{2}=\mathrm{ci}_{13}=348289723$ & $\mathrm{cr}_{2}=\mathrm{cr}_{13}=+4.726253940487881460103682325904548126770$ \\
\hline \multirow[t]{5}{*}{ Error»O $\left(\mathrm{h}^{12}\right)$} & $\mathrm{ci}_{3}=\mathrm{ci}_{12}=-1126407423$ & $\mathrm{cr}_{3}=\mathrm{cr}_{12}=-15.28522712554613596280262946929613596280$ \\
\hline & $\mathrm{ci}_{4}=\mathrm{ci}_{11}=3371637557$ & $\mathrm{cr}_{4}=\mathrm{cr}_{11}=+45.75275765362787932232376676821121265566$ \\
\hline & $\mathrm{ci}_{5}=\mathrm{ci}_{10}=-5718293865$ & $\mathrm{cr}_{5}=\mathrm{cr}_{10}=-77.59663041313431938431938431938431938432$ \\
\hline & $\mathrm{ci}_{6}=\mathrm{ci}_{9}=6277879038$ & $\mathrm{cr}_{6}=\mathrm{cr}_{9}=+85.19014079911215327881994548661215327882$ \\
\hline & $\begin{array}{l}\mathrm{ci}_{7}=\mathrm{ci}_{8}=-2674103430 \\
\text { num }=7\end{array}$ & $\mathrm{cr}_{7}=\mathrm{cr}_{8}=-36.28729485454745871412538079204745871413$ \\
\hline Points $(N)=15$ & $\mathrm{den}=416988000$ & \\
\hline Segments $(N-1)=14$ & $\mathrm{ci}_{2}=\mathrm{ci}_{14}=329062237$ & $\mathrm{cr}_{2}=\mathrm{cr}_{14}=+5.523985483994743254002513261772521031780$ \\
\hline \multirow[t]{7}{*}{ Error»O $\left(\mathrm{h}^{14}\right)$} & $\mathrm{ci}_{3}=\mathrm{ci}_{13}=-1497122214$ & $\mathrm{cr}_{3}=\mathrm{cr}_{13}=-25.13227118766007654896543785432674321563$ \\
\hline & $\mathrm{ci}_{4}=\mathrm{ci}_{12}=6058248882$ & $\mathrm{cr}_{4}=\mathrm{cr}_{12}=+101.7001500618722840945063167285389507612$ \\
\hline & $\mathrm{ci}_{5}=\mathrm{ci}_{11}=-16159538710$ & $\mathrm{cr}_{5}=\mathrm{cr}_{11}=-271.2710460972498009535046572083609120646$ \\
\hline & $\mathrm{ci}_{6}=\mathrm{ci}_{10}=32215733235$ & $\mathrm{cr}_{6}=\mathrm{cr}_{10}=+540.8072477984977984977984977984977984978$ \\
\hline & $\mathrm{ci}_{7}=\mathrm{ci}_{9}=-47966447844$ & $\mathrm{cr}_{7}=\mathrm{cr}_{9}=-805.2153417076750410083743417076750410084$ \\
\hline & $\mathrm{ci}_{8}=54874104828$ & $\mathrm{cr}_{8}=+921.1745512964401853290742179631068519957$ \\
\hline & num $=5$ & \\
\hline Segments $(\mathrm{N}-1)=15$ & $\mathrm{ci}_{2}=\mathrm{ci}_{15}=181146041$ & $\mathrm{cr}_{2}=\mathrm{cr}_{15}=+5.259634135628136744208172779601351029922$ \\
\hline \multirow[t]{6}{*}{ Error»O $\left(\mathrm{h}^{14}\right)$} & $\mathrm{ci}_{3}=\mathrm{ci}_{14}=-737951959$ & $\mathrm{cr}_{3}=\mathrm{cr}_{14}=-21.42667481212054314732886161457590029019$ \\
\hline & $\mathrm{ci}_{4}=\mathrm{ci}_{13}=2671853466$ & $\mathrm{cr}_{4}=\mathrm{cr}_{13}=+77.57813318796159197944912230626516340802$ \\
\hline & $\mathrm{ci}_{5}=\mathrm{ci}_{12}=-6013831334$ & $\mathrm{cr}_{5}=\mathrm{cr}_{12}=-174.6135460405479936729936729936729936730$ \\
\hline & $\mathrm{ci}_{6}=\mathrm{ci}_{11}=9451804423$ & $\mathrm{cr}_{6}=\mathrm{cr}_{11}=+274.4362113135655267351695923124494553066$ \\
\hline & $\mathrm{ci}_{7}=\mathrm{ci}_{10}=-9336416457$ & $\mathrm{cr}_{7}=\mathrm{cr}_{10}=-271.0858842434072623804766661909519052376$ \\
\hline & $\mathrm{ci}_{8}=\mathrm{ci}_{9}=4041701868$ & $\mathrm{cr}_{8}=\mathrm{cr}_{9}=+117.3521264589205437419723134008848294563$ \\
\hline
\end{tabular}

The Table 3 and 4 shows of coefficients for higher order open integration formulas. Figure 2 shows the generation of rules in Maple $12.0 \AA$.

Newton-cotes semi-closed or semi-open integration equation: In the semi-open integration formulas, the last $\left(\mathrm{y}_{\mathrm{N}}\right)$ point does not appear in equation. In the semi-closed integration formulas, the first $\left(\mathrm{y}_{1}\right)$ point does not appear in equation (Zhang et al., 2009). The rule for a double interval is obtained by fitting a zerodegree polynomial to three discrete points. When $\mathrm{N}$ is odd, the semi-closed or semi-open integration formulas are same as open rules. The upper limit of integration is $\mathrm{x}_{3}=\mathrm{x}_{1}+2 \mathrm{~h}$, then the Integral (I) have the formula:

$$
\mathrm{I}=2 \mathrm{~h}\left(\mathrm{y}_{2}\right) \quad \text { or } \quad \mathrm{I}=2 \mathrm{hy}_{2} \quad \text { Error } \approx \mathrm{O}\left(\mathrm{h}^{2}\right)
$$


restart:

for $\mathrm{N}$ from 3 by 1 to 105 do

$\operatorname{assign}(\mathrm{x}, \operatorname{array}(1 . . \mathrm{N}-2))$ : $\operatorname{assign}(\mathrm{y}, \operatorname{array}(1 . . \mathrm{N}-2))$ :

for $\mathrm{j}$ from 1 by 1 to $\mathrm{N}-2$ do $x[\mathrm{j}]:=0+\mathrm{j} * 1$ : od:

$\mathrm{p}:=\operatorname{sort}($ factor $($ int $($ interp $(\mathrm{x}, \mathrm{y}, \mathrm{xx}), \mathrm{xx}=0 . . \mathrm{x}[\mathrm{N}-2]+1)))$ :

$\mathrm{p} 1:=\operatorname{sort}\left(\operatorname{simplify}\left(\mathrm{p}^{*} \mathrm{~h}\right)\right)$;

p2:=evalf (sort $\left.\left(\operatorname{expand}\left(\mathrm{p}^{*} \mathrm{~h}\right)\right), 40\right)$ :

for $\mathrm{j}$ from 1 by 1 to N-2 do print (coeff $(\mathrm{p} 2, \mathrm{y}[\mathrm{j}]))$; od: od:

Fig. 2: Verification Newton-cotes open integration formulas with maple $12.0 \AA$. with number of points $\mathrm{N}=3 . .105$

For three intervals, the semi-open rule is obtained by fitting a first-degree polynomial to four discrete points. The upper limit of integration is $\mathrm{x}_{4}=\mathrm{x}_{1}+3 \mathrm{~h}$, then the Integral (I) have the formula:

$\mathrm{I}=\frac{3 \mathrm{~h}}{4}\left(\mathrm{y}_{1}+3 \mathrm{y}_{3}\right) \quad$ or $\quad \mathrm{I}=0.75 \mathrm{hy}_{1}+2.25 \mathrm{hy}_{3} \quad$ Error $\approx \mathrm{O}\left(\mathrm{h}^{3}\right)$

For $\mathrm{N}=5$, the rule is obtained by fitting a seconddegree polynomial to four equally spaced discrete points, same of the open formula. The upper limit of integration is $\mathrm{x}_{5}$, then:

$\mathrm{I}=\frac{4 \mathrm{~h}}{3}\left(2 \mathrm{y}_{2}-\mathrm{y}_{3}+2 \mathrm{y}_{4}\right)$ or $\quad$ Error $\approx \mathrm{O}\left(\mathrm{h}^{4}\right)$

$\mathrm{I}=2.6666666666666666666666666666666666666667 \mathrm{hy}_{2}$

-1.3333333333333333333333333333333333333333 hy $_{3}$

$+2.6666666666666666666666666666666666666667 \mathrm{hy}_{4}$

For $\mathrm{N}=6$, the rule is obtained by fitting a thirddegree polynomial to five equally spaced discrete points. The upper limit of integration is $\mathrm{x}_{6}$, then:

$$
\begin{aligned}
\mathrm{I}= & \frac{5 \mathrm{~h}}{144}\left(19 \mathrm{y}_{1}-10 \mathrm{y}_{2}+120 \mathrm{y}_{3}-70 \mathrm{y}_{4}+85 \mathrm{y}_{5}\right) \text { or Error } \approx \mathrm{O}\left(\mathrm{h}^{5}\right) \\
\mathrm{I} & =0.6597222222222222222222222222222222222222 \mathrm{hy}_{1} \\
& -0.3472222222222222222222222222222222222222 \mathrm{hy}_{2} \\
& +4.166666666666666666666666666666666666667 \mathrm{hy}_{3} \\
& -2.430555555555555555555555555555555555556 \mathrm{hy}_{4} \\
& +2.951388888888888888888888888888888888889 \mathrm{hy}_{5}
\end{aligned}
$$

Generally, have the semi-open formulas, where $\mathrm{N}$ is number of points:

$$
\begin{aligned}
& \mathrm{I}=\frac{\text { num }}{\operatorname{den}} \mathrm{h}\left(\mathrm{ci}_{1} \mathrm{y}_{1}+\mathrm{ci}_{2} \mathrm{y}_{2}+\mathrm{ci}_{3} \mathrm{y}_{3}+\mathrm{ci}_{4} \mathrm{y}_{4}+\ldots\right. \\
& \left.+\mathrm{ci}_{\mathrm{N}-2} \mathrm{y}_{\mathrm{N}-2}+\mathrm{ci}_{\mathrm{N}-1} \mathrm{y}_{\mathrm{N}-1}\right)
\end{aligned}
$$

restart:

for $\mathrm{N}$ from 3 by 1 to 105 do

$\operatorname{assign}(\mathrm{x}, \operatorname{array}(1 . . \mathrm{N}-1))$ : $\operatorname{assign}(\mathrm{y}, \operatorname{array}(1 . . \mathrm{N}-1))$ :

for $j$ from 1 by 1 to $N-1$ do $x[j]:=0+(j-1) * 1$ : od:

$\mathrm{p}:=\operatorname{sort}($ factor $($ int $($ interp $(\mathrm{x}, \mathrm{y}, \mathrm{xx}), \mathrm{xx}=0 . . \mathrm{x}[\mathrm{N}-1]+1)))$ :

$\mathrm{p} 1:=\operatorname{sort}(\operatorname{simplify}(\mathrm{p} * \mathrm{~h}))$;

p2:= evalf (sort (expand $\left.\left.\left(p^{*} h\right)\right), 40\right)$ :

for $\mathrm{j}$ from 2 by 1 to $\mathrm{N}-1$ do print (coeff (p2, y[j])); od:

od;

Fig. 3: Verification Newton-cotes semi-open integration formulas with maple $12.0 \AA$. with $\mathrm{N}=3 . .105$

The Table 5 and 6 shows of coefficients for higher order open integration formulas. Figure 3 shows the generation of rules in Maple 12.0®.

The semi-closed integration formulas are same as semi-open rules. For examples, $\mathrm{N}=3$ :

$$
\mathrm{I}=2 \mathrm{~h}\left(\mathrm{y}_{2}\right) \quad \text { or } \quad \mathrm{I}=2 \mathrm{hy}_{2} \quad \text { Error } \approx \mathrm{O}\left(\mathrm{h}^{2}\right)
$$

For $\mathrm{N}=4$ :

$\mathrm{I}=\frac{3 \mathrm{~h}}{4}\left(\mathrm{y}_{2}+3 \mathrm{y}_{4}\right) \quad$ or $\quad \mathrm{I}=0.75 \mathrm{hy}_{2}+2.25 \mathrm{hy}_{4} \quad$ Error $\approx \mathrm{O}\left(\mathrm{h}^{3}\right)$

Generally, have the semi-closed formulas, where $\mathrm{N}$ is number of points, ci are integer coefficients and $\mathrm{cr}$ are real coefficients:

$$
\begin{aligned}
\mathrm{I}= & \frac{\operatorname{num}}{\operatorname{den}} \mathrm{h}\left(\mathrm{ci}_{2} \mathrm{y}_{2}+\mathrm{ci}_{3} \mathrm{y}_{3}+\mathrm{ci}_{4} \mathrm{y}_{4}+\ldots\right. \\
& \left.+\mathrm{ci}_{\mathrm{N}-2} \mathrm{y}_{\mathrm{N}-2}+\mathrm{ci}_{\mathrm{N}-1} \mathrm{y}_{\mathrm{N}-1}+\mathrm{ci}_{\mathrm{N}} \mathrm{y}_{\mathrm{N}}\right)
\end{aligned}
$$

or

$$
\mathrm{I}=\mathrm{h}\left(\begin{array}{l}
\mathrm{cr}_{2} \mathrm{y}_{2}+\mathrm{cr}_{3} \mathrm{y}_{3}+\ldots \\
+\mathrm{cr}_{\mathrm{N}-2} \mathrm{y}_{\mathrm{N}-2}+\mathrm{cr}_{\mathrm{N}-1} \mathrm{y}_{\mathrm{N}-1}+\mathrm{cr}_{\mathrm{N}} \mathrm{y}_{\mathrm{N}}
\end{array}\right)
$$

The semi-open or semi-closed rules can be used on type of improper integral-that is, one with a lower limit of $-\infty$ or an upper limit of $+\infty$. Such integrals usually can be evaluated by making a change of variable that transforms the infinite range to one that is finite (Choi et al., 2003). The following identity serves this purpose and works for any function that decreases toward zero at least as fast $1 / x^{2}$ as $x$ approaches infinity:

$$
\int_{a}^{b} f(x) d x=\int_{1 / b}^{1 / a} \frac{1}{w^{2}} f\left(\frac{1}{w}\right) d w
$$


J. Math. \& Stat., 6 (2): 193-204, 2010

Table 4: Newton-cotes open integration formulas with points $=17.23$

\begin{tabular}{lll}
\hline Rule & Integer coefficient & Real coefficient \\
\hline Points $(\mathrm{N})=17$ & num $=16$ & \\
Segments $(\mathrm{N}-1)=16$ & $\mathrm{den}=1915538625$ & \\
Error»O $\left(\mathrm{h}^{16}\right)$ & $\mathrm{ci}_{2}=\mathrm{ci}_{16}=722204696$ & $\mathrm{cr}_{2}=\mathrm{cr}_{16}=+6.032389524904516086173934498449489631147$ \\
& $\mathrm{ci}_{3}=\mathrm{ci}_{15}=-3892087348$ & $\mathrm{cr}_{3}=\mathrm{cr}_{15}=-32.50960161035646044464386616062101070919$ \\
& $\mathrm{ci}_{4}=\mathrm{ci}_{14}=18150263624$ & $\mathrm{cr}_{4}=\mathrm{cr}_{14}=+151.6044699876516454999700149611966190449$ \\
& $\mathrm{ci}_{5}=\mathrm{ci}_{13}=-57468376538$ & $\mathrm{cr}_{5}=\mathrm{cr}_{13}=-480.0185246110607662635881330766692318721$ \\
& $\mathrm{ci}_{6}=\mathrm{ci}_{12}=137035461016$ & $\mathrm{cr}_{6}=\mathrm{cr}_{12}=+1144.621856035923055323408057094124113524$ \\
& $\mathrm{ci}_{7}=\mathrm{ci}_{11}=-249560348012$ & $\mathrm{cr}_{7}=\mathrm{cr}_{11}=-2084.513210059650976758560532810973728081$ \\
& $\mathrm{ci}_{8}=\mathrm{ci}_{10}=355819203336$ & $\mathrm{cr}_{8}=\mathrm{cr}_{10}=+2972.066017920155486293052430618568184706$
\end{tabular}

Points $(\mathrm{N})=18$

Segments $(\mathrm{N}-1)=17$ Error»O $\left(h^{16}\right)$

Points $(\mathrm{N})=19$

Segments $(\mathrm{N}-1)=18$ Error»O $\left(\mathrm{h}^{18}\right)$

Points $(\mathrm{N})=20$

Segments $(\mathrm{N}-1)=19$ Error»O $\left(h^{18}\right)$

Points $(\mathrm{N})=21$ Segments $(\mathrm{N}-1)=20$ Error»O $\left(h^{20}\right)$

Points $(\mathrm{N})=22$

Segments $(\mathrm{N}-1)=21$ Error»O $\left(\mathrm{h}^{20}\right)$ num $=17$

den $=62768369664000$

$\mathrm{ci}_{2}=\mathrm{ci}_{17}=35310023$

$\mathrm{ci}_{3}=\mathrm{ci}_{16}=265553865$

$\mathrm{ci}_{4}=\mathrm{ci}_{15}=-232936065$

$\mathrm{ci}_{5}=\mathrm{ci}_{14}=1047777585$

$\mathrm{ci}_{6}=\mathrm{ci}_{13}=-1562840685$

$\mathrm{ci}_{7}=\mathrm{ci}_{12}=2461884669$

$\mathrm{ci}_{8}=\mathrm{ci}_{11}=-2000332805$

$\mathrm{ci}_{9}=\mathrm{ci}_{10}=1018807605$

num $=9$

den $=9529520000$

$\mathrm{ci}_{2}=\mathrm{ci}_{18}=6912171129$

$\mathrm{ci}_{3}=\mathrm{ci}_{17}=-43087461474$

$\mathrm{ci}_{4}=\mathrm{ci}_{16}=227788759000$

$\mathrm{ci}_{5}=\mathrm{ci}_{15}=-834322842510$

$\mathrm{ci}_{6}=\mathrm{ci}_{14}=2317367615100$

$\mathrm{ci}_{7}=\mathrm{ci}_{13}=-4988390746282$

$\mathrm{ci}_{8}=\mathrm{ci}_{12}=8524579147752$

$\mathrm{ci}_{9}=\mathrm{ci}_{11}=-11696802277350$

$\mathrm{ci}_{10}=12990970309270$

num $=19$

den $=64023737057280000$

$\mathrm{ci}_{2}=\mathrm{ci}_{19}=21156441141866149$

$\mathrm{ci}_{3}=\mathrm{ci}_{18}=-121972899306097215$

$\mathrm{ci}_{4}=\mathrm{ci}_{17}=596043470364791516$

$\mathrm{ci}_{5}=\mathrm{ci}_{16}=-1967193294708433100$

$\mathrm{ci}_{6}=\mathrm{ci}_{15}=4792224378449610000$

$\mathrm{ci}_{7}=\mathrm{ci}_{14}=-8635040534820624232$

$\mathrm{ci}_{8}=\mathrm{ci}_{13}=11419549616838153340$

$\mathrm{ci}_{9}=\mathrm{ci}_{12}=-10248543211438519308$

$\mathrm{ci}_{10}=\mathrm{ci}_{11}=4175787902007892850$ num $=5$

$\operatorname{den}=1247337455364$

$\mathrm{ci}_{2}=\mathrm{ci}_{20}=1749481500626$

$\mathrm{ci}_{3}=\mathrm{ci}_{19}=-12389954060697$

$\mathrm{ci}_{4}=\mathrm{ci}_{18}=73278572831682$

$\mathrm{ci}_{5}=\mathrm{ci}_{17}=-304672055470086$

$\mathrm{ci}_{6}=\mathrm{ci}_{16}=966316491145704$

$\mathrm{ci}_{7}=\mathrm{ci}_{15}=-2400158698258188$

$\mathrm{ci}_{8}=\mathrm{ci}_{14}=4782407754794376$

$\mathrm{ci}_{9}=\mathrm{ci}_{13}=-7751977518223986$

$\mathrm{ci}_{10}=\mathrm{ci}_{12}=10322815990097148$

$\mathrm{ci}_{11}=-11349750778891702$

num $=7$

den $=136216903680000$

$\mathrm{ci}_{2}=\mathrm{ci}_{21}=131721567613331$

$\mathrm{ci}_{3}=\mathrm{ci}_{20}=-871503959599375$

$\mathrm{ci}_{4}=\mathrm{ci}_{19}=4813298466509865$

$\mathrm{ci}_{5}=\mathrm{ci}_{18}=-18345435138969285$

$\mathrm{ci}_{6}=\mathrm{ci}_{17}=52322284124735964$

$\mathrm{ci}_{7}=\mathrm{ci}_{16}=-113381582504747148$

$\mathrm{ci}_{8}=\mathrm{ci}_{15}=188254898608060740$

$\mathrm{ci}_{9}=\mathrm{ci}_{14}=-234658964587522740$ $\mathrm{cr}_{2}=\mathrm{cr}_{17}=+5.776080028330126933659781984296975478633$ $\mathrm{cr}_{3}=\mathrm{cr}_{16}=-28.40482276561302211999412175779018812529$

$\mathrm{cr}_{4}=\mathrm{cr}_{15}=+120.7857119531877634590652668254079188823$ $\mathrm{cr}_{5}=\mathrm{cr}_{14}=-336.0196952465624741067036040581014125988$ $\mathrm{cr}_{6}=\mathrm{cr}_{13}=+675.9477281475243925812984454959763601739$ $\mathrm{cr}_{7}=\mathrm{cr}_{12}=-957.7617592663299065036554013626324031968$ $\mathrm{cr}_{8}=\mathrm{cr}_{11}=+902.0728860329482302483018973318796951954$ $\mathrm{cr}_{9}=\mathrm{cr}_{10}=-373.8961288834851104919722644590369458094$

$\mathrm{cr}_{2}=\mathrm{cr}_{18}=+6.528087475654597503336999135318463049555$ $\mathrm{cr}_{3}=\mathrm{cr}_{17}=-40.69325141937894038734374868828650341255$ $\mathrm{cr}_{4}=\mathrm{cr}_{16}=+215.1313844768676701449810693508172499769$ $\mathrm{cr}_{5}=\mathrm{cr}_{15}=-787.9626237827298751668499567659231524778$ $\mathrm{cr}_{6}=\mathrm{cr}_{14}=+2188.600111642559121550718189373651558526$ $\mathrm{cr}_{7}=\mathrm{cr}_{13}=-4711.204417068016017595849528622637866335$ $\mathrm{cr}_{8}=\mathrm{cr}_{12}=+8050.899975000629622478361974160293488025$ $\mathrm{cr}_{9}=\mathrm{cr}_{11}=-11046.85445816263568364408700543154324667$ $\mathrm{cr}_{10}=+12269.11038367409901023346401497662001864$

$\mathrm{cr}_{2}=\mathrm{cr}_{19}=+6.278489825356881835928412120617397789995$ $\mathrm{cr}_{3}=\mathrm{cr}_{18}=-36.19727921758873480653772631674903694823$ $\mathrm{cr}_{4}=\mathrm{cr}_{17}=+176.8848001921393224671383928914101476954$ $\mathrm{cr}_{5}=\mathrm{cr}_{16}=-583.7939851280550748211558677720451071767$ $\mathrm{cr}_{6}=\mathrm{cr}_{15}=+1422.164143731269979555752198142487591601$ $\mathrm{cr}_{7}=\mathrm{cr}_{14}=-2562.577220614401768069350071286648386624$ $\mathrm{cr}_{8}=\mathrm{cr}_{13}=+3388.921870115258482018911113388410480087$ $\mathrm{cr}_{9}=\mathrm{cr}_{12}=-3041.408233373194120867756294149073483029$ $\mathrm{cr}_{10}=\mathrm{cr}_{11}=+1239.227414469215032687069842981590396606$

$\mathrm{cr}_{2}=\mathrm{cr}_{20}=+7.012863652504780777619044396407280048932$ $\mathrm{cr}_{3}=\mathrm{cr}_{19}=-49.66560575654863142437769429566798286866$ $\mathrm{cr}_{4}=\mathrm{cr}_{18}=+293.7399679475580661266111534587995997771$ $\mathrm{cr}_{5}=\mathrm{cr}_{17}=-1221.289612365468958918554481275996132751$ $\mathrm{cr}_{6}=\mathrm{cr}_{16}=+3873.516693458515541474781051053405349250$ $\mathrm{cr}_{7}=\mathrm{cr}_{15}=-9621.128139528889042469653401485523548124$ $\mathrm{cr}_{8}=\mathrm{cr}_{14}=+19170.46479374246868509030813848777421471$ $\mathrm{cr}_{9}=\mathrm{cr}_{13}=-31074.09901341330118168988869965816949939$ $\mathrm{cr}_{10}=\mathrm{cr}_{12}=+41379.40356759641848596208767827772804682$ $\mathrm{cr}_{11}=-45495.91103066651548985786557791751465496$

$\mathrm{cr}_{2}=\mathrm{cr}_{21}=+6.768990840221960035672424410814503693761$ $\mathrm{cr}_{3}=\mathrm{cr}_{20}=-44.78539412059277986959452226480090257180$ $\mathrm{cr}_{4}=\mathrm{cr}_{19}=+247.3488117504173693459774874248558459085$ $\mathrm{cr}_{5}=\mathrm{cr}_{18}=-942.7467700665400633484726702605959571904$ $\mathrm{cr}_{6}=\mathrm{cr}_{17}=+2688.770475458451912449167189879264182670$ $\mathrm{cr}_{7}=\mathrm{cr}_{16}=-5826.5241397478668833908641667929593626188$ $\mathrm{cr}_{8}=\mathrm{cr}_{15}=+9674.161243248905274191591432303506606912$ $\mathrm{cr}_{9}=\mathrm{cr}_{14}=-12058.80259891588794040631066559859129519$ 
Table 4: Continued

\begin{tabular}{|c|c|c|}
\hline & $\mathrm{ci}_{10}=\mathrm{ci}_{13}=203086455887932170$ & $\mathrm{cr}_{10}=\mathrm{cr}_{13}=+10436.33464577312869074899236470443900784$ \\
\hline & $\begin{array}{l}\mathrm{ci}_{11}=\mathrm{ci}_{12}=-81146847108493522 \\
\text { num }=11\end{array}$ & $\mathrm{cr}_{11}=\mathrm{cr}_{12}=-4170.025264220237589238381372669298365893$ \\
\hline Points $(\mathrm{N})=23$ & $\operatorname{den}=136073176948800000$ & \\
\hline Segments $(N-1)=22$ & $\mathrm{ci}_{2}=\mathrm{ci}_{22}=92630057200320343$ & $\mathrm{cr}_{2}=\mathrm{cr}_{22}=+7.488107884678219107763794023251961361867$ \\
\hline Error»O $\left(\mathrm{h}^{22}\right)$ & $\mathrm{ci}_{3}=\mathrm{ci}_{21}=-734938747634165690$ & $\mathrm{cr}_{3}=\mathrm{cr}_{21}=-59.41160782200078205336853449914283228910$ \\
\hline & $\mathrm{ci}_{4}=\mathrm{ci}_{20}=4810326117267632170$ & $\mathrm{cr}_{4}=\mathrm{cr}_{20}=+388.8612618330624445981208858188398831456$ \\
\hline & $\mathrm{ci}_{5}=\mathrm{ci}_{19}=-22377003329240798370$ & $\mathrm{cr}_{5}=\mathrm{cr}_{19}=-1808.931356943816102313267694473241452700$ \\
\hline & $\mathrm{ci}_{6}=\mathrm{ci}_{18}=79806119136903527115$ & $\mathrm{cr}_{6}=\mathrm{cr}_{18}=+6451.435398147956012301934773154146760206$ \\
\hline & $\mathrm{ci}_{7}=\mathrm{ci}_{17}=-224650147742325069072$ & $\mathrm{cr}_{7}=\mathrm{cr}_{17}=-18160.46101499777111663885000033407847909$ \\
\hline & $\mathrm{ci}_{8}=\mathrm{ci}_{16}=511409287186029264120$ & $\mathrm{cr}_{8}=\mathrm{cr}_{16}=+41341.74188615452911701408934393529574465$ \\
\hline & $\mathrm{ci}_{9}=\mathrm{ci}_{15}=-956236430810735088960$ & $\mathrm{cr}_{9}=\mathrm{cr}_{15}=-77301.05943565865461982799972793457733459$ \\
\hline & $\mathrm{ci}_{10}=\mathrm{ci}_{14}=1484914688541488498910$ & $\mathrm{cr}_{10}=\mathrm{cr}_{14}=+120038.8051504254972433824004775105596634$ \\
\hline & $\mathrm{ci}_{11}=\mathrm{ci}_{13}=-1928478457946599022420$ & $\mathrm{cr}_{11}=\mathrm{cr}_{13}=-155895.9929729168011329179167912379773400$ \\
\hline & $\mathrm{ci}_{12}=2103160001429187403708$ & $\mathrm{cr}_{12}=+170017.0491677866414346941869480738468518$ \\
\hline
\end{tabular}

Table 5: Newton-cotes semi-open integration formulas with points $=4,6,8,10,12,14,16$

\begin{tabular}{|c|c|c|c|}
\hline Rule & Integer coefficient & Real coefficient & \\
\hline & num $=3$ & $\operatorname{den}=4$ & \\
\hline Points $(N)=4$ & $\mathrm{ci}_{1}=1$ & & $\mathrm{cr}_{1}=+0.7500000000000000000000000000000000000000$ \\
\hline Segments $(N-1)=3$ & $\mathrm{ci}_{2}=0$ & & $\mathrm{cr}_{3}=+0.000000000000000000000000000000000000000$ \\
\hline Error»O $\left(h^{3}\right)$ & $\mathrm{ci}_{3}=3$ & $d e n=144$ & $\mathrm{cr}_{3}=+2.250000000000000000000000000000000000000$ \\
\hline Points $(N)=6$ & $\begin{array}{l}\mathrm{num}=\mathrm{J} \\
\mathrm{ci}_{1}=19\end{array}$ & $\operatorname{den}=144$ & $\mathrm{cr}_{1}=+0.6597222222222222222222222222222222222222$ \\
\hline Segments $(N-1)=5$ & $\mathrm{ci}_{2}=-10$ & & $\mathrm{cr}_{2}=-0.3472222222222222222222222222222222222222$ \\
\hline \multirow[t]{4}{*}{ Error»O $\left(h^{5}\right)$} & $\mathrm{ci}_{3}=120$ & & $\mathrm{cr}_{3}=+4.166666666666666666666666666666666666666667$ \\
\hline & $\mathrm{ci}_{4}=-70$ & & $\mathrm{cr}_{4}=-2.4305555555555555555555555555555555555555556$ \\
\hline & $\mathrm{ci}_{5}=85$ & & $\mathrm{cr}_{5}=+2.951388888888888888888888888888888888888889$ \\
\hline & num $=7$ & $\operatorname{den}=8640$ & \\
\hline Points $(N)=8$ & $\mathrm{ci}_{1}=751$ & & $\mathrm{cr}_{1}=+0.6084490740740740740740740740740740740741$ \\
\hline Segments $(\mathrm{N}-1)=7$ & $\mathrm{ci}_{2}=-840$ & & $\mathrm{cr}_{2}=-0.68055555555555555555555555555555555555556$ \\
\hline \multirow[t]{6}{*}{ Error»O $\left(h^{7}\right)$} & $\mathrm{ci}_{3}=8547$ & & $\mathrm{cr}_{3}=+6.9246527777777777777777777777777777777777778$ \\
\hline & $\mathrm{ci}_{4}=-11648$ & & $\mathrm{cr}_{4}=-9.437037037037037037037037037037037037037$ \\
\hline & $\mathrm{ci}_{5}=14637$ & & $\mathrm{cr}_{5}=+11.85868055555555555555555555555555555556$ \\
\hline & $\mathrm{ci}_{6}=-7224$ & & $\mathrm{cr}_{6}=-5.852777777777777777777777777777777777778$ \\
\hline & $\mathrm{ci}_{7}=4417$ & & $\mathrm{cr}_{7}=+3.578587962962962962962962962962962962963$ \\
\hline & num $=9$ & $\operatorname{den}=44800$ & \\
\hline Points $(\mathrm{N})=10$ & $\mathrm{ci}_{1}=2857$ & & $\mathrm{cr}_{1}=+0.5739508928571428571428571428571428571429$ \\
\hline Segments $(N-1)=9$ & $\mathrm{ci}_{2}=-4986$ & & $\mathrm{cr}_{2}=-1.001651785714285714285714285714285714286$ \\
\hline \multirow[t]{8}{*}{ Error»O $\left(\mathrm{h}^{9}\right)$} & $\mathrm{ci}_{3}=51966$ & & $\mathrm{cr}_{3}=+10.43959821428571428571428571428571428571$ \\
\hline & $\mathrm{ci}_{4}=-110322$ & & $\mathrm{cr}_{4}=-22.16290178571428571428571428571428571429$ \\
\hline & $\mathrm{ci}_{5}=182880$ & & $\mathrm{cr}_{5}=+36.73928571428571428571428571428571428571$ \\
\hline & $\mathrm{ci}_{6}=-177102$ & & $\mathrm{cr}_{6}=-35.57852678571428571428571428571428571429$ \\
\hline & $\mathrm{ci}_{7}=129666$ & & $\mathrm{cr}_{7}=+26.04897321428571428571428571428571428571$ \\
\hline & $\mathrm{ci}_{8}=-50886$ & & $\mathrm{cr}_{8}=-10.22263392857142857142857142857142857143$ \\
\hline & $\mathrm{ci}_{9}=20727$ & & $\mathrm{cr}_{9}=+4.16390625000000000000000000000000000000$ \\
\hline & num $=11$ & $\operatorname{den}=43545600$ & \\
\hline Points $(\mathrm{N})=12$ & $\mathrm{ci}_{1}=2171465$ & & $\mathrm{cr}_{1}=+0.5485310800631981187536743092298647854203$ \\
\hline Segments $(N-1)=11$ & $\mathrm{ci}_{2}=-5199788$ & & $\mathrm{cr}_{2}=-1.313511996619635508524397413286302175191$ \\
\hline \multirow[t]{10}{*}{ Error»O $\left(\mathrm{h}^{11}\right)$} & $\mathrm{ci}_{3}=58096731$ & & $\mathrm{cr}_{3}=+14.67574315200617283950617283950617283951$ \\
\hline & $\mathrm{ci}_{4}=-166532520$ & & $\mathrm{cr}_{4}=-42.06757330246913580246913580246913580247$ \\
\hline & $\mathrm{ci}_{5}=353493954$ & & $\mathrm{cr}_{5}=+89.29566922949735449735449735449735449735$ \\
\hline & $\mathrm{ci}_{6}=-493861632$ & & $\mathrm{cr}_{6}=-124.7537742504409171075837742504409171076$ \\
\hline & $\mathrm{ci}_{7}=509355198$ & & $\mathrm{cr}_{7}=+128.6675847387566137566137566137566137566$ \\
\hline & $\mathrm{ci}_{8}=-363089496$ & & $\mathrm{cr}_{8}=-91.71958719135802469135802469135802469136$ \\
\hline & $\mathrm{ci}_{9}=191759205$ & & $\mathrm{cr}_{9}=+48.44005490795855379188712522045855379189$ \\
\hline & $\mathrm{ci}_{1}=-61333844$ & & $\mathrm{cr}_{10}=-15.49346625146972369194591416813639035861$ \\
\hline & $\mathrm{ci}_{11}=18686327$ & & $\mathrm{cr}_{11}=+4.720329884075543797766019988242210464433$ \\
\hline & num $=13$ & $\operatorname{den}=201180672000$ & \\
\hline Points $(\mathrm{N})=14$ & $\mathrm{ci}_{1}=8181904909$ & & $\mathrm{cr}_{1}=+0.5287026967332130195886809643423400037157$ \\
\hline Segments $(N-1)=13$ & $\mathrm{ci}_{2}=-25042017078$ & & $\mathrm{cr}_{2}=-1.618178420310674774960489246203531917818$ \\
\hline \multirow{3}{*}{ Error»O $\left(\mathrm{h}^{13}\right)$} & $\mathrm{ci}_{3}=303460165164$ & & $\mathrm{cr}_{3}=+19.60915085884592333005031417729830428243$ \\
\hline & $\mathrm{ci}_{4}=-1091975193010$ & & $\mathrm{cr}_{4}=-70.56183562767898498718604538710358816179$ \\
\hline & $\mathrm{ci}_{5}=2849201218305$ & & $\mathrm{cr}_{5}=+184.1112044698061253120776930300739824549$ \\
\hline
\end{tabular}


J. Math. \& Stat., 6 (2): 193-204, 2010

Table 5: Continued

Points $(\mathrm{N})=16$ Segments $(\mathrm{N}-1)=15$ Error»O $\left(h^{15}\right)$

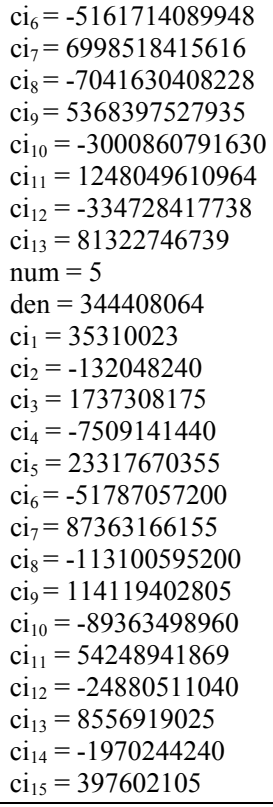

$\mathrm{cr}_{6}=-333.542395013592558235415378272521129664$ $\mathrm{cr}_{7}=+452.2339969269413713858158302602747047191$ $\mathrm{cr}_{8}=-455.0198306672521702283607045511807416569$ $\mathrm{cr}_{9}=+346.8979756820525979752170228360704551181$ $\mathrm{cr}_{10}=-193.9112236944411836938291964746991202018$ $\mathrm{cr}_{11}=+80.64713563801993861517671041480565290089$ $\mathrm{cr}_{12}=-21.62965948634469219786680104140421600739$ $\mathrm{cr}_{13}=+5.254956637221094479692363290246888130486$

$\mathrm{cr}_{1}=+0.5126189931487783050283050283050283050283$ $\mathrm{cr}_{2}=-1.917031768454759526188097616669045240474$ $\mathrm{cr}_{3}=+25.22165356441828261024689596118167546739$ $\mathrm{cr}_{4}=-109.0151803181937110508539079967651396223$ $\mathrm{cr}_{5}=+338.5180661013790896603396603396603396603$ $\mathrm{cr}_{6}=-751.8270129702886399314970743542172113601$ $\mathrm{cr}_{7}=+1268.308952182373987619523333809048094762$ $\mathrm{cr}_{8}=-1641.956258027686599115170543741972313401$ $\mathrm{cr}_{9}=+1656.746962884701793741972313400884829456$ $\mathrm{cr}_{10}=-1297.349108527261429047143332857618571904$ $\mathrm{cr}_{11}=+787.5678234554926100685029256457827886399$ $\mathrm{cr}_{12}=-361.2068595467032967032967032967032967033$ $\mathrm{cr}_{13}=+124.2264615645004177370248798820227391656$ $\mathrm{cr}_{14}=-28.60334071620343941772513201084629656058$ $\mathrm{cr}_{15}=+5.772253128776915049236477807906379334951$

Table 6: Newton-cotes semi-open integration formulas with points $=18,20,22$

\section{Rule}

Points $(\mathrm{N})=18$ Segments $(\mathrm{N}-1)=17$ Error»O $\left(h^{17}\right)$

Points $(\mathrm{N})=20$ Segments $(\mathrm{N}-1)=19$ Error» $\mathrm{O}\left(\mathrm{h}^{19}\right)$ Integer coefficient num $=17$

den $=1883051089920000$

$\mathrm{ci}_{1}=55294720874657$

$\mathrm{ci}_{2}=-244912369711442$

$\mathrm{ci}_{3}=3489029300972250$

$\mathrm{ci}_{4}=-17585886834501250$

$\mathrm{ci}_{5}=63416259440780110$

$\mathrm{ci}_{6}=-166654158061846266$

$\mathrm{ci}_{7}=336711046842479186$

$\mathrm{ci}_{8}=-532651057910098250$

$\mathrm{ci}_{9}=670227439244428800$

$\mathrm{ci}_{10}=-673987225218482870$

$\mathrm{ci}_{11}=542720673660231086$

$\mathrm{ci}_{12}=-347616418702275846$

$\mathrm{ci}_{13}=175509574710531250$

$\mathrm{ci}_{14}=-68185176240903550$

$\mathrm{ci}_{15}=20014523360265510$

$\mathrm{ci}_{16}=-4031052737981102$

$\mathrm{ci}_{17}=695097885157727$

num $=19$

den $=2688996956405760000$

$\mathrm{ci}_{1}=69028763155644023$

$\mathrm{ci}_{2}=-353947208843214156$

$\mathrm{ci}_{3}=5438538991957452489$

$\mathrm{ci}_{4}=-31293644979684279096$

$\mathrm{ci}_{5}=128605896878516520180$

$\mathrm{ci}_{6}=-390165018822674369064$

$\mathrm{ci}_{7}=918778256758909425228$

$\mathrm{ci}_{8}=-1717150274758012947672$

$\mathrm{ci}_{9}=2590121803284253347498$

$\mathrm{ci}_{10}=-3180795372743080898560$

$\mathrm{ci}_{11}=3195943710049002658134$

$\mathrm{ci}_{12}=-2627210173545633198888$

$\mathrm{ci}_{13}=1761071043128578083252$

$\mathrm{ci}_{14}=-954110145180024206808$

$\mathrm{ci}_{15}=412501439151154330380$
Real coefficient

$\mathrm{cr}_{1}+0.4991953005954313348171740294021305191205$

$\mathrm{cr}_{2}=-2.211044781196774423415002486137112827295$

$\mathrm{cr}_{3}=+31.49861330583873805806676177046547416918$

$\mathrm{cr}_{4}=-158.7636563802537840385521896397851718251$

$\mathrm{cr}_{5}=+572.5157518371225552605531294537761322006$

$\mathrm{cr}_{6}=-1504.537344853319677900117714932529747345$

$\mathrm{cr}_{7}=+3039.794207901884222712274226089628793920$

$\mathrm{cr}_{8}=-4808.721352778786240060168999028493443543$

$\mathrm{cr}_{9}=+6050.747389779716168605057493946382835272$

$\mathrm{cr}_{10}=-6084.690367695219580800443160819410084548$

$\mathrm{cr}_{11}=+4899.628853201162359464231524784140892313$

$\mathrm{cr}_{12}=-3138.246832267173976985071561791138510715$

$\mathrm{cr}_{13}=+1584.483175231209421948555179007853904973$

$\mathrm{cr}_{14}=-615.5690635800040216043210605232945033063$

$\mathrm{cr}_{15}=+180.6891480246395236371261503536635811768$

$\mathrm{cr}_{16}=-36.39194757513992347706890622822427643121$

$\mathrm{cr}_{17}=+6.275275328925558268476956013699105997754$

$\mathrm{cr}_{1}=+0.4877456245656414838932399711855527103437$

$\mathrm{cr}_{2}=-2.500931416824664874149907360722550996191$

$\mathrm{cr}_{3}=+38.42780134095441222912798927464052773436$

$\mathrm{cr}_{4}=-221.1156294534241283897454235960008639451$

$\mathrm{cr}_{5}=+908.7076260428078658921584440557461864750$

$\mathrm{cr}_{6}=-2756.840367547146254441527874975328030624$

$\mathrm{cr}_{7}=+6491.932553822166738924756753801952128196$

$\mathrm{cr}_{8}=-12133.09488606171610139955772962061897389$

$\mathrm{cr}_{9}=+18301.36480637014593133263836498834201619$

$\mathrm{cr}_{10}=-22474.96485191227391420225755605998238030$

$\mathrm{cr}_{11}=+22582.00045421255508488746450211900589583$

$\mathrm{cr}_{12}=-18563.42498955016870428622513715810293701$

$\mathrm{cr}_{13}=+12443.43164455182698901301793847701099491$

$\mathrm{cr}_{14}=-6741.581731892818002066630144404464008849$ $\mathrm{cr}_{15}=+2914.665754902132920269066509970278885252$ 
Table 6: Continued

\begin{tabular}{|c|c|}
\hline $\begin{array}{l}\text { Points }(\mathrm{N})=22 \\
\text { Segments }(\mathrm{N}-1)=21 \\
\text { Error»O }\left(\mathrm{h}^{21}\right)\end{array}$ & $\begin{array}{l}\mathrm{ci}_{16}=-138949589112759712968 \\
\mathrm{ci}_{17}=35595226518134779191 \\
\mathrm{ci}_{18}=-6365379507657675444 \\
\mathrm{ci}_{19}=957599291114022281 \\
\mathrm{num}=7 \\
\mathrm{den}=14983859404800000 \\
\mathrm{ci}_{1}=1022779523247467 \\
\mathrm{ci}_{2}=-5966218027482930 \\
\mathrm{ci}_{3}=98462673861087480 \\
\mathrm{ci}_{4}=-636505825186027230 \\
\mathrm{ci}_{5}=2937368924847356265 \\
\mathrm{ci}_{6}=-10101722474707772328 \\
\mathrm{ci}_{7}=27170960245549634640 \\
\mathrm{ci}_{8}=-58577829795256960440 \\
\mathrm{ci}_{9}=103027050438855916590 \\
\mathrm{ci}_{10}=-149446538576972018620 \\
\mathrm{ci}_{11}=180038500415174725632 \\
\mathrm{ci}_{12}=-180712201906578844740 \\
\mathrm{ci}_{13}=151179046691155956690 \\
\mathrm{ci}_{14}=-105098354746771143240 \\
\mathrm{ci}_{15}=60350973167958502320 \\
\mathrm{ci}_{16}=-28329147803950914648 \\
\mathrm{ci}_{17}=10710818043854933655 \\
\mathrm{ci}_{18}=-3183966521788733730 \\
\mathrm{ci}_{19}=723790940733103880 \\
\mathrm{ci}_{20}=-116321026020880590 \\
\mathrm{ci}_{21}=15512151960713877\end{array}$ \\
\hline
\end{tabular}

Table 7: Set of points

\begin{tabular}{lllllll}
\hline$x$ & 0.0 & 0.1 & 0.2 & 0.3 & 0.4 & 0.5 \\
$y=f(x)$ & 1.0000 & 1.1052 & 1.2214 & 1.3499 & 1.4918 & 1.6487 \\
\hline
\end{tabular}

For $a b>0$. Therefore, it can be used only when a is positive and $b$ is $\infty$ or when $a$ is $-\infty$ and $b$ is negative. For cases where the limits are from $-\infty$ to $\infty$, the integral can be implemented in three steps. For example:

$$
\int_{-\infty}^{\infty} f(x) d x=\int_{-\infty}^{-A} f(x) d x+\int_{-A}^{A} f(x) d x+\int_{A}^{\infty} f(x) d x
$$

Where, $\mathrm{A}$ is a positive number. One problem with using Eq. 21 to evaluate an integral is that the transformed function will be singular at one of the limits (Sun and $\mathrm{Wu}, 2005)$. The semi-open or semi-closed integration formulas can be used to circumvent this dilemma as they allow evaluation of integral without employing data at the end points of integration interval.

Analysis of error: Let's solve the example problem presented in Eq. 23 using Simpson's 1/3 rule (Sun and $\mathrm{Wu}, 2008)$. The exact solution is $\mathrm{I}_{\text {exact }}=\mathrm{e}^{0.8}-\mathrm{e}^{0.0}=$ 1.2255409:

$$
I=\int_{0}^{0.8} e^{x} d x
$$

Solving the problems for two increments of $h=0.4$, the minimum permissible number of increments for Simpson's $1 / 3$ rule and one interval yields: $\mathrm{cr}_{16}=-981.7944147736185256780396842594561188171$ $\mathrm{cr}_{17}=+251.5098807506824695028041084827997123780$ $\mathrm{cr}_{18}=-44.97670045977028151661604579808898573441$ $\mathrm{cr}_{19}=+6.766235449922523319821652091802950500339$

$\mathrm{cr}_{1}+0.4778112547184455679924133079916924555258$ $\mathrm{cr}_{2}=-2.787234254146951324175841749019345416756$ $\mathrm{cr}_{3}=+45.99874427591187804896400625362066397811$ $\mathrm{cr}_{4}=-297.35601862861057816537368368567355333910$ $\mathrm{cr}_{5}=+1372.248759044328713574769806959153989832$ $\mathrm{cr}_{6}=-4719.215217696328173705208737223935647803$ $\mathrm{cr}_{7}=+12693.44009313908338147729814982840594999$ $\mathrm{cr}_{8}=-27365.76722252499515658028820321249254545$ $\mathrm{cr}_{9}=+48131.08115796670025959799374211490732740$ $\mathrm{cr}_{10}=-69816.843696736988909256746845558022582227$ $\mathrm{cr}_{11}=+84108.47091254089177076793175864383294724$ $\mathrm{cr}_{12}=-84423.20360673035518924412058295396319601$ $\mathrm{cr}_{13}=+70626.21840265571689075329677241793763043$ $\mathrm{cr}_{14}=-49098.73106468978837117819030111459044755$ $\mathrm{cr}_{15}=+28194.12547613585548957753125006150618309$ $\mathrm{cr}_{16}=-13234.50983290264692006301759503279345666$ $\mathrm{cr}_{17}=+5003.766004569320689372409667099014129692$ $\mathrm{cr}_{18}=-1487.451600445568010859823841371125356490$ $\mathrm{cr}_{19}=+338.1329501469220272645360159432774124584$ $\mathrm{cr}_{20}=-54.34161921496169122944278842463475168232$ $\mathrm{cr}_{21}=+7.246802094940405603664837718806196149286$

$$
\begin{gathered}
\mathrm{I}(\mathrm{h}=0.4)=\frac{0.4}{3}[1+4(1.49182470)+2.22554093] \\
=1.22571196
\end{gathered}
$$

Breaking the total range of integration into four increments of $\mathrm{h}=0.2$ and two intervals and applying the composite rule yields:

$$
\begin{aligned}
\mathrm{I}(\mathrm{h}=0.2)= & \frac{0.2}{3}[1+4(1.22140276)+2(1.49182470) \\
& +4(1.82211880)+2,225540938] \\
& =1.22555177
\end{aligned}
$$

Error $=|1.22555177-1.2255409|=0.0000108$

The global error of Simpson's $1 / 3$ rule is $O\left(h^{4}\right)$. Thus, for successive increment havings:

$$
\begin{aligned}
& \text { Ratio }=\frac{0.0001710}{0.0000108}=15.775 \\
& \text { Ratio }=\frac{\text { Error }(\mathrm{h})}{\text { Error }(\mathrm{h} / 2)}=\frac{\mathrm{O}\left[(\mathrm{h})^{4}\right]}{\mathrm{O}\left[(\mathrm{h} / 2)^{4}\right]}=2^{4}=16 \cong 15.775
\end{aligned}
$$

Mixing rules: To estimate the error of integration in discrete function, we can apply different rules or mix several integration formulas of Newton-cotes (Berriochoa et al., 2007). The difference in the result of each formula provides an approximation of the error. For example, calculate the integral of the points in Table 7. 
Using trapezoidal rule:

$$
\begin{aligned}
& I=\frac{0.1}{2}[1.0000+2(1.1052)+2(1.2214) \\
&+2(1.3499)+2(1.4918)+1.6487]=0.6493
\end{aligned}
$$

Using Simpson's 1/3 and 3/8 rule:

$$
\begin{aligned}
\mathrm{I}= & \frac{0.1}{3}[1.0000+2(1.1052)+1.2214]+ \\
& \frac{3(0.1)}{8}[1.2214+3(1.3499)+3(1.4918)+1.6487] \\
& =0.6487
\end{aligned}
$$

Using closed rule with $\mathrm{N}=6$ :

$$
\begin{aligned}
& I=\frac{5(0.1)}{288}[19(1.0000)+75(1.1052)+50(1.2214)+ \\
& 50 .(1.3499)+75(1.4918)+19(1.6487)]=0.6487
\end{aligned}
$$

Using open rule with $\mathrm{N}=6$ :

$$
\begin{aligned}
I=\frac{5(0.1)}{24}[0(1.0000)+11(1.1052)+1.2214+ \\
1.3499+11(1.4918)+0(1.6487)]=0.6487
\end{aligned}
$$

Then, estimation of the error is $|0.6487-0.6493|=$ 0.0006 and the best result is 0.6487 .

\section{CONCLUSION}

Newton-Cotes Integration is very important. To improve the error, formulas of high order should be applied. To estimate the error of integration for set of points proposed to apply different rules or mix various integration formulas. The difference in the result of each formula provides an approximation of the error of integration. And we can choose the best result as the average of all values or the value that appeared more times using the various formulas of integration. Computational routines to generate Newton-Cotes integration rules were presented with number of points until one hundred. The equations of Newton-Cotes shown in the article can be used in many scientific applications.

\section{ACKNOWLEDGMENT}

The researchers thank the generous support of the Pontificia Universidade Catolica de Minas GeraisPUCMINAS.

\section{REFERENCES}

Berriochoa, E., A . Cachafeiro and F. Marcellan, 2007. A new numerical quadrature formula on the unit circle. Numer. Algorithms, 44: 391-401. DOI: 10.1007/s11075-007-9121-3

Choi, S.H., J.H. Park and Y.Y. Park, 2003. A study on the average case error of composite Newton-cotes quadratures. J. Applied Math. Comput., 12: 107117. DOI: $10.1007 / \mathrm{BF} 02936185$

Espelid, T.O., 2003. Doubly adaptive quadrature routines based on Newton-cotes rules. BIT Numer. Mathe., 43: 319-337. DOI: 10.1023/A:1026087703168

Kalogiratou, Z. and T.E. Simos, 2003. Newton-cotes formulae for long-time integration. J. Comput. Applied Math., 158: 75-82. DOI: 10.1016/S03770427(03)00479-5

Sermutlu, E. and H.T. Eyyubog'lu, 2007. A new quadrature routine for improper and oscillatory integrals. Applied Math. Comput., 189: 452-461. DOI: $10.1016 /$ j.amc.2006.08.176

Sermutlu, E., 2005. Comparison of Newton-cotes and Gaussian methods of quadrature. Applied Math. Compu., 171: 1048-1057. DOI: 10.1016/j.amc.2005.01.102

Simos, T.E., 2008. High-order closed Newton-cotes trigonometrically-fitted formulae for long-time integration of orbital problems. Comput. Phys Commun., 178: 199-207. DOI: 10.1016/j.cpc.2007.08.016

Simos, T.E., 2009. High order closed Newton-cotes trigonometrically-fitted formulae for the numerical solution of the schrodinger equation. Applied Math. Comput., 209: 137-151. DOI: 10.1016/j.amc.2008.06.020

Sun, W. and J. Wu, 2005. Newton-cotes formulae for the numerical evaluation of certain hypersingular integrals. Computing, 75: 297-309. DOI: 10.1007/s00607-005-0131-5

Sun, W. and J. Wu, 2008. The superconvergence of Newton-cotes rules for the hadamard finite-part integral on an interval. Numer. Math., 109: 143-165. DOI: 10.1007/s00211-007-0125-7

Witteveen, J.A.S., A. Loeven and H. Bijl, 2009. An adaptive stochastic finite elements approach based on Newton-cotes quadrature in simplex elements. Comput. Fluids, 38: 1270-1288. DOI: 10.1016/j.compfluid.2008.12.002

Zhang, X. J. Wu and D. Yu, 2009. The superconvergence of composite Newton-cotes rules for hadamard finite-part integral on a circle. Computing, 85: 219-244. DOI: 10.1007/s00607009-0048-5 